\title{
LOS ESTUDIOS PSICOLÓGICOS DE LA SUSTENTABILIDAD HÍDRICA. APLICACIONES AL SISTEMA TARIFARIO DE CONSUMO
}

\section{THE PSYCHOLOGICAL STUDIES OF THE SUSTAINABILITY. APPLICATIONS TO THE TARIFF SYSTEM OF CONSUMER}

\author{
Cruz García Lirios* \\ José Marcos Bustos Aguayo**
}

\section{RESUMEN}

El propósito del presente artículo es exponer la historia, las estructuras y las aplicaciones de los estudios psicológicos en torno a la sustentabilidad del diseño de sistemas tarifarios del servicio de agua potable. Para tal propósito, se plantean dos tendencias del estado de la cuestión: conservacionismo y comunitarismo. Se analizan las investigaciones conservacionistas en cuatro estructuras: validez, covarianza, predicción y disturbio. Finalmente, se ofrece una síntesis de las investigaciones conservacionistas para su aplicación en el diseño de sistemas de cotización del abasto y consumo de agua.

PALABRAS CLAVE: PSICOLOGÍA * SUSTENTABILIDAD * RECURSOS HÍDRICOS * CONSERVACIONISMO $*$ COMUNITARISMO * AHORRO * DISPENDIO * TARIFAS

\section{ABSTRACT}

The purpose of the present article is expose the history, the structures and the applications of the psychological studies around the sustainability of the design of tariff systems of the service of potable water. We are considered two tendencies of the state of the question: conservationism and communitarism. The conservationists' investigations are analyzed in four structures: validity, covariance, prediction and disturbance. Finally, it provides a synthesis of the conservationists' investigations for their application in the design of quote systems in the supply and water consumption.

KEYWORDS: PSYCHOLOGY * SUSTAINABILITY * WATER * CONSERVATIONISM * COMMUNITARIANISM * SAVINGS * WASTE * RATES 


\section{INTRODUCCIÓN}

Las situaciones ambientales y los estilos de vida sustentables han sido estudiados a partir de su estructura, desde la sociología de Weber, Parsons, Merton, Giddens, Powell y DiMagio. El término estructura alude a la interrelación entre sentimientos, valores, motivos, intenciones y comportamientos deliberados, planificados y sistemáticos, limitados por niveles de necesidad, satisfacción, información, capacidades y recursos. Precisamente, es en este sentido que el término estructura cobra relevancia en relación con otro término fundamental: la sustentabilidad.

Si la estructura es el resultado de las interrelaciones entre los individuos y junto a ellos sus valores, sentimientos, motivos, intenciones y comportamientos, entonces la sustentabilidad sería una estructura de las necesidades de las generaciones actuales en función de la disponibilidad de recursos y de las capacidades de las generaciones futuras.

Sin restar importancia a las causas ambientales, económicas, sociales, políticas, culturales o tecnológicas que impiden el desarrollo sustentable, se expondrán los factores psicológicos que inciden en el Desarrollo Sustentable. Para tal propósito, se elaboró una revisión de los estudios psicológicos en torno a la sustentabilidad, principalmente se revisaron estructuras de validez, covarianzas, predicciones $y$ disturbios en torno al dispendio $y$ ahorro de recursos naturales.

Antes de iniciar la exposición de los estudios psicológicos de la sustentabilidad, es preponderante establecer que las subdisciplinas psicológicas han contribuido en mayor o menor medida en la explicación de los factores que impiden a la humanidad desarrollarse sustentablemente. Sin embargo, los estudios psicológicos de la comunidad y la conservación han influido sustancialmente en las investigaciones disciplinarias actuales. Tales estudios hegemónicos plantean estructuras de sentimientos en el caso de los comunitarios, así como de valores, motivos, intenciones y comportamientos en el caso de los conservacionistas con el fin de explicar las situaciones ambientales y los estilos de vida sustentables.

También es importante establecer que los estudios psicológicos de la sustentabilidad han tenido más influencia de los estudios psicológicos conservacionistas que comunitarios. Esta influencia conservacionista es evidente si se consideran los hallazgos de validez, covarianzas, predicciones y disturbios. Desde sus inicios, los estudios psicológicos conservacionistas, han aplicado los modelos matemáticos estadísticos para demostrar estructuras de estilos de vida sustentables a partir de las interrelaciones entre constructos tales como: normas, valores, creencias, percepciones, actitudes, motivos, conocimientos, competencias, intenciones y comportamientos. Desde estos estudios psicológicos conservacionistas se plantea una relación directa e indirecta entre las situaciones ambientales y los estilos de vida. Dicha relación esta mediada por los constructos psicológicos mencionados.

Las Psicologías de la Sustentabilidad son once subdisciplinas que fueron confiabilizando, validando y modelizando variables en torno a las situaciones ambientales y los estilos sustentables. Los conceptos esenciales de las once subdisciplinas (ver tabla 1) se articulan en una estructura de factores temporales, espaciales, situacionales, económicos, culturales y psicológicos, predictores del comportamiento que extrae, procesa, distribuye, consume, reutiliza y recicla los recursos naturales en función de sus necesidades actuales $y$ las necesidades de sus generaciones posteriores. 
TABLA 1

LA PSICOLOGÍA DE LA SUSTENTABILIDAD

\begin{tabular}{llllc}
\hline AÑO & AUTOR & PSICOLOGÍA & $\begin{array}{c}\text { EXPLICACIÓN } \\
\text { (ERKL̈̈RUNG) }\end{array}$ & $\begin{array}{c}\text { COMPRENSIÓN } \\
\text { (VERSTEHEN) }\end{array}$ \\
\hline 1879 & Wunt & Étnica & & $\checkmark$ \\
1910 & McDougal & Social & $\checkmark$ \\
1911 & Hellpach & Ambiental & & $\checkmark$ \\
1935 & Muchow & Arquitectónica & & $\checkmark$ \\
1953 & Skinner & Conductista & $\checkmark$ & \\
1968 & Barker & Ecológica & $\checkmark$ & \\
1974 & Everett & Conservacionista & $\checkmark$ & \\
1974 & Sarason & Comunitaria & & \\
1980 & Berk, Cooley, LaCivita, Parker, Sredl $y$ Brewer & Hídrica & $\checkmark$ & \\
1989 & Crawford $y$ Anderson & Evolucionista & $\checkmark$ & \\
1990 & Seligman & Positiva & $\checkmark$ & \\
\hline
\end{tabular}

Fuente: Elaboración propia.

En 1854, Seattle (Ce-atl/líder-agua), jefe máximo de los Pieles Rojas, respondía a la oferta de reubicación propuesta por Washington:

¿Cómo se puede comprar o vender el firmamento, ni aún el calor de la tierra? Si no somos dueños de la frescura del aire ni del fulgor de las aguas, ¿cómo podrían ustedes comprarlas? Cada parcela de esta tierra es sagrada para mi pueblo. Cada brillante mata de pino, cada grano de arena en las playas, cada gota de rocío en los oscuros bosques, cada altozano, es sagrado a la memoria y al pasado de mi pueblo. La savia que circula por las venas de los árboles lleva consigo las memorias de los Pieles Rojas. Somos parte de la tierra y ella es parte de nosotros. Las flores perfumadas son nuestras hermanas; el venado, el caballo, el águila; estos son nuestros hermanos. Las escarpadas peñas, los húmedos prados todos pertenecemos a la misma familia.

Y sobre el agua decía:

El agua cristalina que corre por ríos y arroyuelos no es solamente agua, sino también representa la sangre de nuestros antepasados. El murmullo del agua es la voz del padre de mi padre (...) y cada reflejo en las claras aguas de los lagos cuenta los sucesos y memorias de las vidas de nuestras gentes.

Las ciencias sociales tienen en sus cimientos, palabras tales como: tribu, campo, espíritu, alma, sentimiento y recuerdo, a partir de las cuales Dilthey las llamó "Ciencias del Espíritu" (Geisteswissenschaften) para diferenciarlas de las "Ciencias de la Naturaleza" (Naturwissenschaften). Posteriormente, Weber definió a las ciencias sociales como comprensivas (Verstehen) y a las ciencias naturales como explicativas (Erklärung). Por ello, el inicio de la Psicología es étnico, es decir, buscaba comprender.

En 1879, Wunt publicó un libro que llamó Psicología de los pueblos, a partir del cual se derivan las variables de "espíritu" en torno a la relación entre las especies y la naturaleza. Wunt consideraba que los símbolos y significados no estaban separados, $y$ el pueblo percibía los recursos naturales de tal manera que eran un elemento del pueblo y se fusionaban con el pueblo mismo. La gente 
respetaba el entorno porque era un elemento del pueblo inseparable a su introspección y convivencia. El agua (wasser), la psicología (psychologie) y el pueblo (volks) formaban un solo espíritu (geist).

Para 1910, McDougal, con el nombre de "Psicología Social", registra una publicación y acuña el término de "mente social" para relacionarlo con el de "espíritu de los pueblos" propuesto por Wunt, con el fin de comprender los procesos de identidad en torno a la naturaleza. Es decir, el respeto por los recursos parecía derivarse de ese espíritu o mente propuestos por estas dos aproximaciones. La mente y el espíritu se separan, la naturaleza y la psicología empiezan a separarse.

Un año después, Hellpach propuso el concepto de "ambiente fáctico" y su impacto en la psique, a lo cual llama "psique de lo fáctico". Aquí aparece el entorno relacionado con la psique humana. Una vez separadas la psique, la mente y el espíritu, la naturaleza es percibida $y$ con ello incluida en el mundo de lo real. La naturaleza y la psique, una vez separadas, pueden analizarse dividiendo sus partes en relaciones para después estudiar su variación y con ello, su medición y predicción.

Muchow, en 1935, exportó la psicología arquitectónica a los Estados Unidos para explicar el impacto del diseño y edificaciones sobre el comportamiento humano. La sociedad demandaba espacios, diseños y edificaciones que los aseguraran de la incertidumbre que vivieron durante la Primera y Segunda Guerra Mundial. Se trataba de una psicología arquitectónica que a partir de los valores de seguridad, orden y progreso promovía los valores de la modernidad urbana avocada al control de los recursos naturales. Entre la naturaleza y la humanidad se encuentra la arquitectura sustentada en una psicología de la certidumbre y la seguridad.

En 1953, Skinner realizó análisis experimentales de las "relaciones contingentes" entre el comportamiento condicionado y los "estímulos discriminativos" que anteceden a los reforzadores. Este autor descubre que el entorno y el comportamiento humano parecían estar mediados por procesos psicológicos.
Para 1968, Barker hizo inteligible la dimensión del "espacio ecológico" como el determinante del comportamiento humano. El cuidado de los recursos estaba determinado por el espacio individual. A mayor espacio personal le correspondía una alta probabilidad de conservación de los recursos. La psicología modera la relación entre la naturaleza y la humanidad, entre la disponibilidad de los recursos y las necesidades.

Por su parte, en 1974, Everett refiere al constructo esencial de "conducta ecológica responsable" en relación al desarrollo de las economías norteamericanas, asiáticas y europeas del Pacífico y Atlántico, cuyas necesidades energéticas las llevan a incrementar la explotación de los recursos naturales. La psicología de la conservación sintetiza los conceptos abstractos de sus antecesoras para explicar el consumismo y los efectos de la contaminación atmosférica en la salud. La conservación de los recursos era la resultante de la relación entorno-comportamiento. La psicología parece haber abandonado la "comprensión" para avocarse a la explicación de la naturaleza con base en las necesidades humanas. No obstante, en el mismo año, Sarason propone el concepto de "Sentimiento de Comunidad" para comprender el efecto de las enfermedades en los asentamientos humanos. Se trata de una aproximación sanitaria en la que la insalubridad, derivada de la escasez y abasto irregular de agua, tiene un efecto directo sobre la dinámica comunitaria.

En 1980, Berk, Cooley, LaCivita, Parker, Sredl y Brewer publican un artículo sobre la reducción del consumo de agua en una situación de escasez. Los estudios psicológicos de la sustentabilidad, para explicar el impacto de la escasez, desabasto e insalubridad del agua sobre el comportamiento humano, recuperan el conocimiento de las subdisciplinas expuestas para demostrar la premisa de conservación a partir de los costos y beneficios en el consumo de agua.

Algunos años después, en 1989, Crawford y Anderson desde una aproximación evolucionista, relacionaron el comportamiento anti-ambiental con los mecanismos psicológicos básicos, a partir de los cuales las especies compiten por la 
obtención de recursos y con ello participan en la selección de especies adaptándose al entorno. En esta carrera por la supervivencia, las especies animales $y$ vegetales, adoptan estilos de vida que los llevan, instintiva y psíquicamente, a la competencia y almacenamiento de los recursos. Ambos estilos evolutivos garantizaron la adaptación de la especie a su entorno; sin embargo, el medio ambiente en el que las especies compiten, cambia constantemente $y$ toda adaptación por vía de la competencia es posterior a las exigencias del entorno.

Finalmente, en 1990, Seligman escribe un influyente libro: El optimismo aprendido en el que sostiene que las personas dedicadas, optimistas $y$ atemporales construyen un mundo de felicidad a partir del cual aprenden a reconocer sus necesidades en función de sus motivos, esfuerzos y logros. Incluso, las personas felices transmiten este aprendizaje a sus grupos de pertenencia y referencia mediante los procesos de identidad e influencia social. La psicología de la sustentabilidad adopta los postulados de la psicología positiva para explicar los comportamientos pro-sustentables.

En un contexto de racionalidad de costos $y$ beneficios, las psicologías de la sustentabilidad adoptaron los métodos de la psicología de la conservación y soslayaron los métodos de la psicología de la comunidad. La vertiente conservacionista prosperó y hasta el momento es el paradigma dominante. La vertiente comunitaria parece retornar con los estudios de representación social y sentimiento comunitario. Sin embargo, la vertiente conservacionista se ha radicalizado al plantear una omnisciencia, considerando que el comportamiento es un reflejo codificado genéticamente y que predispone a las personas a afrontar las situaciones ambientales.

Una vez expuesta la historia de la Psicología de la Sustentabilidad, se exponen las Psicologías de la Sustentabilidad Hídrica.

El nacimiento de las Psicologías de la Sustentabilidad Hídrica tiene como fecha de registro el año de 1980, cuando Berk, Cooley, LaCivita, Parker, Sredl y Brewer llevan acabo un estudio sobre la relación causal, directa y significativa, entre la escasez y el ahorro de agua. Transcurridos 31 años de investigación en torno al agua (ver tabla 2), pueden observarse dos líneas de investigación que parten de los planteamientos "comprensivos" y "explicativos". Se trata de una vertiente orientada a la explicación de la sustentabilidad y otra vertiente orientada a su comprensión. 


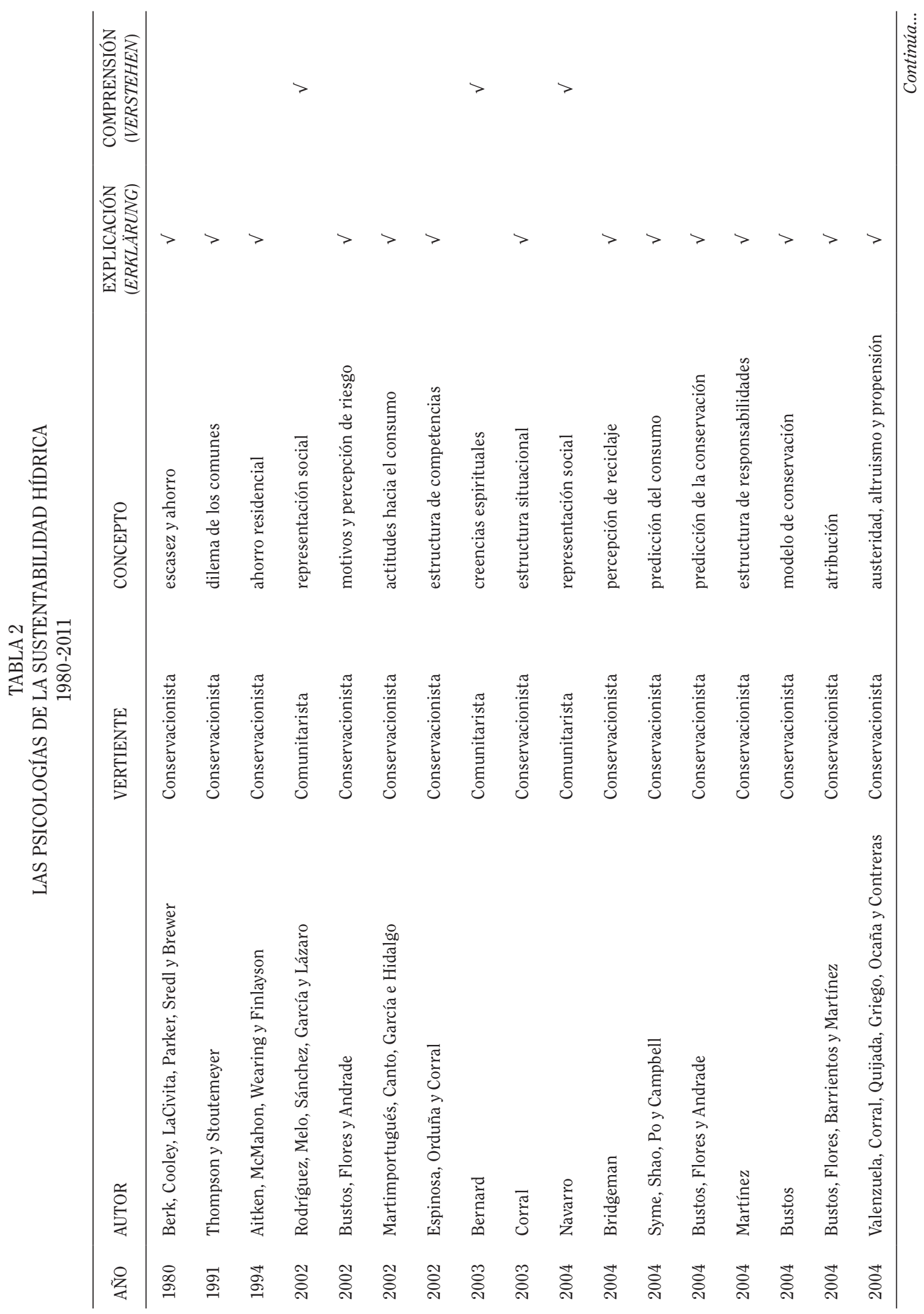




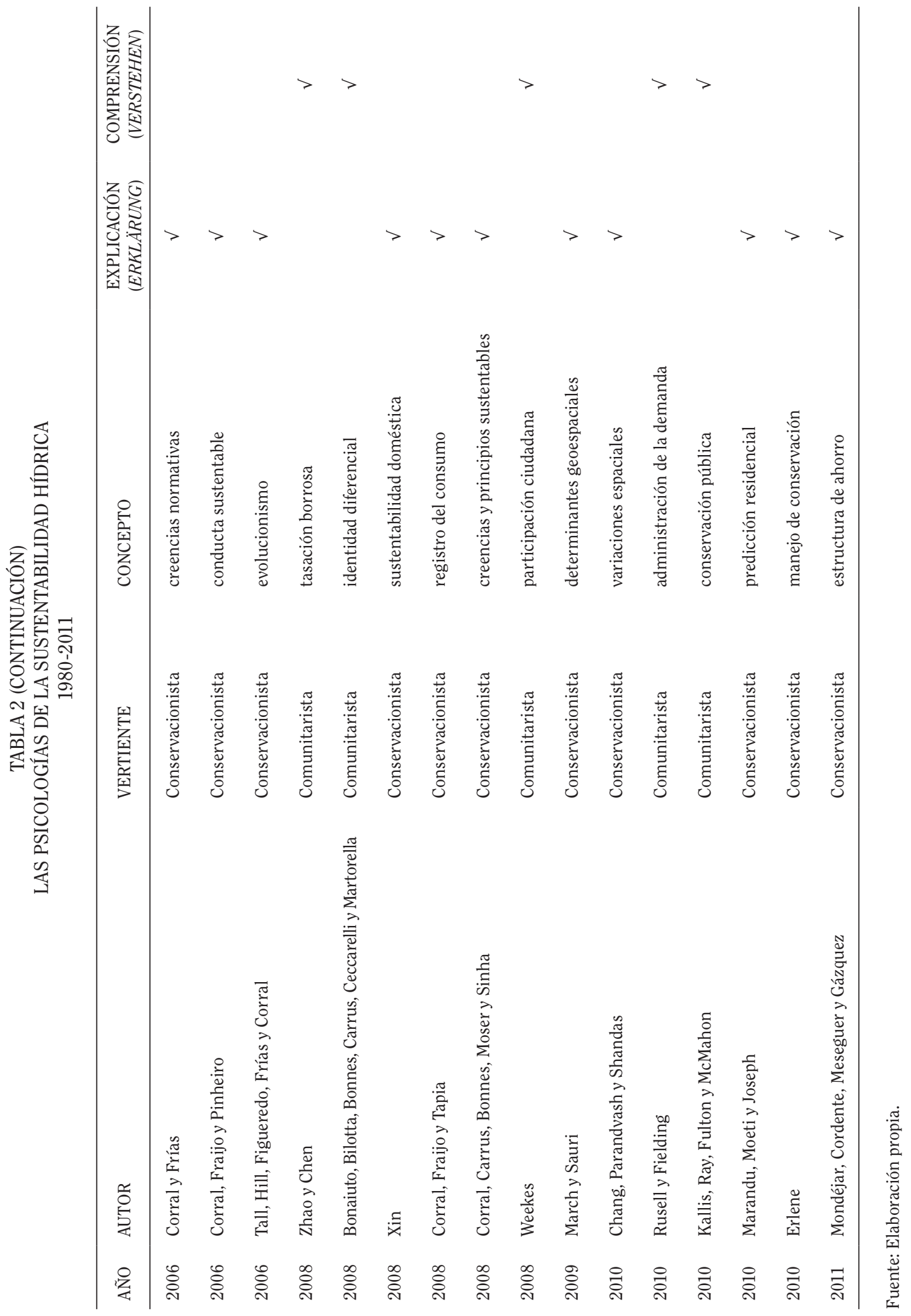


Las psicologías conservacionistas a través de modelos estructurales, buscan la predicción de los comportamientos anti y pro-sustentables. Han demostrado las relaciones indirectas $y$ directas, negativas y positivas, insignificantes $y$ significantes entre los indicadores y los constructos de primer $y$ segundo orden. Hasta el momento, no existe un consenso en la conceptuación, pero si en la operacionalización, medición, análisis y síntesis de dichas variables y sus relaciones entre ellas.

Los estudios psicológicos conservacionistas pueden clasificarse en cuatro estructuras: validez, covariación, predicción y disturbio. Cada una de ellas es ponderada por parámetros que demuestran la convergencia de rasgos cognitivos $y$ conductuales en factores, la asociación entre dimensiones, la predicción de comportamientos $y$ los errores de ponderación (ver figura 1 ).

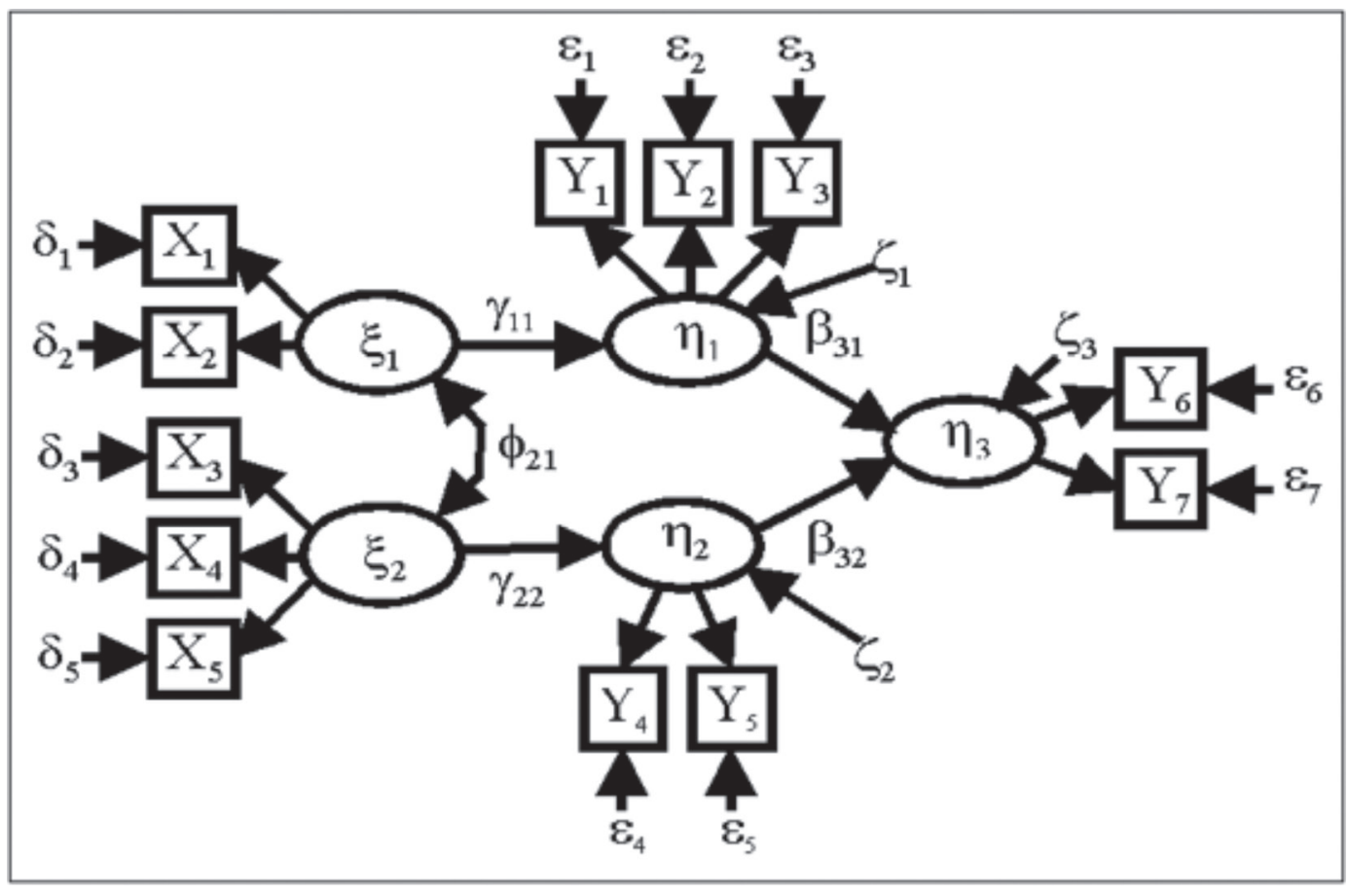

FIGURA 1

NOMENCLATURA DE UNA ESTRUCTURA

Fuente: Kline, (2006).

Las estructuras de validez o covergencia de factores se estiman con pesos factoriales que suponen la correlación entre los rasgos cognitivos-conductuales $(\mathrm{X})$ con factores subyacentes

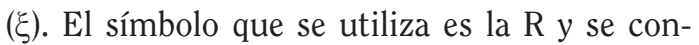
sidera un valor superior a .300, suficiente para demostrar la convergencia de rasgos psicológicos en factores (Auxiliadora y Manera, 2003).

La estructura de covariación es ponderada por asociaciones entre los factores emergentes de la estructura de validez. Se emplea el símbolo phi $(\Phi)$ para denotar un incremento paralelo u opuesto entre los factores subyacentes $(\xi)$. Sin importar el valor de covariación $y$ el signo, la significancia es imprescindible para demostrar una estructura. Una significancia menor a .05 es suficiente para interpretar una asociación trascendente entre los factores (Kline, 1998).

La estructura de predicción establece la relación causal entre un rasgo (X) y un factor $(\xi)$, así como entre rasgos y también entre factores $(\xi \eta)$. El símbolo gamma $(\gamma)$ es designado para las predicciones entre factores exógenos $y$ 
el símbolo beta $(\beta)$ es escogido para la predicción entre factores endógenos. En estos parámetros el nivel de significancia menor a .05 es fundamental para demostrar causas y efectos (Levy, 2003).

La estructura de disturbio o errores de medición calcula la influencia de rasgos y factores no incluidos en un modelo. Se utiliza el símbolo delta $(\delta)$ para los errores de medición de los rasgos de factores exógenos ( $(\xi)$, el símbolo épsilon $(\varepsilon)$ para los errores de medición de los rasgos de factores endógenos ( $\eta$ ) y el símbolo sigma ( $\varsigma$ ) para los errores de los factores endógenos $(\eta)$.

Finalmente, las estructuras son consideradas hipótesis de relaciones que se contrastan con índices de ajuste, los cuales demostrarían la verosimilitud de las relaciones entre rasgos $y$ factores (Kline, 2006). Estas son:

\section{MEDIDAS ABSOLUTAS}

$\diamond \quad$ Parámetro de No Centralidad (Non Central Parameter, NCP)

$\diamond \quad$ Parámetro de No Centralidad de la Escala (Scale Non Central Parameter, SNCP)

$\diamond \quad$ Índice de Bondad de Ajuste (Goodness of Fit Index, GFI)

$\diamond \quad$ Residuo Cuadrático Medio Estandarizado (Standardized Root Mean Residual, SRMR)

$\diamond \quad$ Error de Aproximación Cuadrático Medio (Root Mean Squared Error of Approximation, RMSEA)

$\diamond \quad$ Índice de Validación Cruzada Esperada (Ease Crossed Validation Index, ECVI)

\section{MEDIDAS INCREMENTALES}

$\diamond \quad$ Índice de Bondad de Ajuste Ajustado (Adjust Goodness of Fit Index, AGFI)

$\diamond \quad$ Índice Tucker Lewis (Tucker Lewis Index, TLI)

$\diamond \quad$ Índice de Ajuste Normal (Normed Fit Index, NFI)

$\diamond \quad$ Índice de Ajuste Relativo (Relative Fit Index, RFI) $\diamond \quad$ Indice de Ajuste Incremental (Incremental Fit Index, IFI)

$\diamond \quad$ Indice de Ajuste Comparado (Comparative Fit Index, CFI)

\section{MEDIDAS PARSIMONIOSAS}

$\diamond \quad$ Índice de Ajuste Normado de Parsimonia (Parsimony Normed Fit Index, PNFI)

$\diamond \quad$ Índice de Bondad de Ajuste de Parsimonia (Parsimony Goodness of Fit Index, PGFI)

$\diamond \quad$ Criterio de Información de Akaike (Akaike Information Criterion, AIC)

Cada uno de ellos asume valores cercanos a la unidad para el caso de los índices de ajuste $y$ valores cercanos a cero para los índices residuales. La verosimilitud de una estructura se interpreta a partir de la contrastación de hipótesis donde:

Hipótesis nula: $\sum$ la matriz de relaciones hipotéticas es igual a $\sum$ la matriz de relaciones calculadas.

Hipótesis alterna: $\sum$ la matriz de relaciones hipotéticas es diferente a $\sum$ la matriz de relaciones calculadas.

La aceptación de la hipótesis nula sugiere que las relaciones entre las variables (rasgos y factores psicológicos) del modelo estructural explican y predicen las acciones sustentables. A continuación, se exponen las implicaciones de estos términos con los sistemas tarifarios de consumo de agua.

\section{CONFIGURACIONES CAUSALES DE ACCIONES ANTI Y/O PRO SUSTENTABLES}

Las creencias subyacen como factor de sustentabilidad. El antropocentrismo y el ecocentrismo se configuran por la convergencia de rasgos psicológicos que favorecen o interfieren en el desarrollo sostenible. Las estructuras de creencias implican modelos robustos e índices de ajuste cercanos a la unidad $y$ residuales aproximados al cero (ver tabla 3). 
TABLA 3

ESTRUCTURAS DE VALIDEZ

\begin{tabular}{|c|c|c|c|c|}
\hline AÑO & AUTOR & MUESTRA & ESTIMACIÓN & AJUSTE \\
\hline 1999 & $\begin{array}{l}\text { Bechtel, Corral y } \\
\text { Pinheiro }\end{array}$ & $\begin{array}{l}164 \text { estudiantes } \\
\text { de Estados } \\
\text { Unidos }\end{array}$ & $\begin{array}{l}\text { Establecieron la validez del instrumento que } \\
\text { miden las creencias ambientales en los Estados } \\
\text { Unidos de América }\left(\mathrm{R}_{1}=.70, \mathrm{R}_{2}=.50, \mathrm{R}_{3}=.73 \text {, }\right. \\
\mathrm{R}_{4}=.27, \mathrm{R}_{5}=.71 \text { y } \mathrm{R}_{6}=.80, \mathrm{R}_{7}=.45, \mathrm{R}_{8}=.53, \mathrm{R}_{9} \\
\left.=.65, \mathrm{R}_{10}=.52, \mathrm{R}_{11}=.55, \mathrm{R}_{12}=.89\right)\end{array}$ & $\begin{array}{l}\mathrm{X}^{2}=64.4 ; 53 \mathrm{gl} \\
\mathrm{p}=.14\end{array}$ \\
\hline 1999 & $\begin{array}{l}\text { Bechtel, Corral y } \\
\text { Pinheiro }\end{array}$ & $\begin{array}{l}505 \text { estudiantes } \\
\text { de México }\end{array}$ & $\begin{array}{l}\text { Demostraron la validez del instrumento que } \\
\text { mide las creencias ecológicas }\left(\mathrm{R}_{1}=.58, \mathrm{R}_{2}=.31\right. \\
\mathrm{R}_{3}=.50, \mathrm{R}_{4}=.38, \mathrm{R}_{5}=.41 \text { y } \mathrm{R}_{6}=.33, \mathrm{R}_{7}=.72 \\
\left.\mathrm{R}_{8}=.45, \mathrm{R}_{9}=.43, \mathrm{R}_{10}=.92, \mathrm{R}_{11}=.63, \mathrm{R}_{12}=.23\right)\end{array}$ & $\begin{array}{l}\mathrm{X}^{2}=58.24 ; 51 \\
\mathrm{gl} ; .22\end{array}$ \\
\hline 1999 & $\begin{array}{l}\text { Bechtel, Corral y } \\
\text { Pinheiro }\end{array}$ & $\begin{array}{l}137 \text { estudiantes } \\
\text { de Brasil }\end{array}$ & $\begin{array}{l}\text { Establecieron la validez del instrumento que } \\
\text { mide las creencias del entorno }\left(\mathrm{R}_{1}=.25, \mathrm{R}_{2}=\right. \\
.48, \mathrm{R}_{3}=.59, \mathrm{R}_{4}=.27, \mathrm{R}_{5}=.99 \text { y } \mathrm{R}_{6}=.40, \mathrm{R}_{7}= \\
\left..78, \mathrm{R}_{8}=.33, \mathrm{R}_{9}=.43\right) .\end{array}$ & $\begin{array}{l}\mathrm{X}^{2}=36.44 ; 52 \mathrm{gl} \\
\mathrm{p}=.31\end{array}$ \\
\hline 2002 & Corral & $\begin{array}{l}195 \text { residentes de } \\
\text { México }\end{array}$ & $\begin{array}{l}\text { Demostró la validez de los instrumentos que } \\
\text { miden el nuevo paradigma ambiental (limites } \\
\text { al crecimiento }=.59 \text {, economía de Estado }=.57 \\
\text { y tierra como nave espacial }=.60 \text { ), el paradigma } \\
\text { de la excepción humana (modificación del } \\
\text { ambiente }=.36 \text {, humanidad sobre naturaleza }= \\
.73 \text { y negativa de adaptación }=.71 \text { ) y los motivos } \\
\text { ecológicos (reuso de cartón }=.60 \text {, reuso de } \\
\text { periódico }=.61 \text {, reuso de ropa }=.73 \text { y reuso de } \\
\text { cajas }=.75 \text { ). }\end{array}$ & $\begin{array}{l}\mathrm{X}^{2}=1.61 ; 8 \mathrm{gl} ; \mathrm{p} \\
=.99 \mathrm{NFI}=.99 ; \\
\mathrm{NNFI}=1.08 ; \mathrm{CFI} \\
=1.00\end{array}$ \\
\hline 2002 & Corral & $\begin{array}{l}200 \text { residentes de } \\
\text { Ciudad Obregón, } \\
\text { Sonora, México }\end{array}$ & $\begin{array}{l}\text { Estableció que regar las plantas, lavar trastes } \\
\text { y lavarse los dientes son indicadores }\left(\mathrm{R}_{1}=.53 \text {, }\right. \\
\mathrm{R}_{2}=.76 \text { y } \mathrm{R}_{3}=.75 \text { respectivamente) de las } \\
\text { habilidades. }\end{array}$ & $\begin{array}{l}\mathrm{X}^{2}=43 ; 34 \mathrm{gl} ; \mathrm{p} \\
=.47 \mathrm{NFI}=.95 \\
\mathrm{NNFI}=1 ; \mathrm{CFI}=1\end{array}$ \\
\hline 2002 & Corral & $\begin{array}{l}195 \text { residentes } \\
\text { de Hermosillo, } \\
\text { Sonora, México }\end{array}$ & $\begin{array}{l}\text { Estableció que los motivos para reutilizar ropa, } \\
\text { cajas, vidrio y periódico son indicadores de la } \\
\text { reutilización ( } \mathrm{R}_{1}=.65, \mathrm{R}_{2}=.62, \mathrm{R}_{3}=.75 \text { y } \mathrm{R}_{4}= \\
.59 \text { respectivamente). }\end{array}$ & $\begin{array}{l}\mathrm{X}^{2}=249,7 ; 103 \mathrm{gl} ; \\
\mathrm{p}<.001 \text { IANN }= \\
0,91 ; \mathrm{IAC}=0,93 ; \\
\text { GFI = 1,0; AGFI } \\
=.91 ; \text { RQQMEA } \\
=0,04\end{array}$ \\
\hline 2002 & $\begin{array}{l}\text { Espinosa, Orduña } \\
\text { y Corral }\end{array}$ & $\begin{array}{l}485 \text { residentes } \\
\text { de Hermosillo, } \\
\text { Sonora, México }\end{array}$ & $\begin{array}{l}\text { Demostraron que los motivos son indicadores } \\
\left(\mathrm{R}_{1}=.15\right) \text { de las competencias de ahorro de agua. } \\
\text { Asimismo, bañarse, lavar trastes y lavarse los } \\
\text { dientes son indicadores de las habilidades ( } \mathrm{R}_{1}= \\
.80, \mathrm{R}_{2}=.85 \text { y } \mathrm{R}_{3}=.24 \text { respectivamente). }\end{array}$ & $\begin{array}{l}\mathrm{X}^{2}=271.5 ; 84 \mathrm{gl} \\
\mathrm{p}<.001 \mathrm{NFI}=.90 \\
\mathrm{CFI}=.93 ; \mathrm{RMSEA} \\
=.03\end{array}$ \\
\hline
\end{tabular}




\section{TABLA 3 (CONTINUACIÓN)}

ESTRUCTURAS DE VALIDEZ

\begin{tabular}{|c|c|c|c|c|}
\hline AÑO & AUTOR & MUESTRA & ESTIMACIÓN & AJUSTE \\
\hline 2003 & Corral & $\begin{array}{l}500 \text { residentes } \\
\text { de Hermosillo y } \\
\text { Ciudad Obregón, } \\
\text { Sonora, México }\end{array}$ & $\begin{array}{l}\text { Demostró que lavar platos, regar plantas } y \\
\text { tomar baño de chuveiro son indicadores de las } \\
\text { habilidades }\left(\mathrm{R}_{1}=\mathrm{R}_{2}=.58, \mathrm{R}_{3}=.57 \text { y } \mathrm{R}_{4}=.50\right. \\
\text { respectivamente). }\end{array}$ & $\begin{array}{l}\mathrm{X}^{2}=249,7 ; 103 \\
\text { gl; } \mathrm{p}<.001 \text { IANN } \\
=0,91 ; \mathrm{IAC}=.93 ; \\
\text { GFI = } 1 ; \text { RQQMEA } \\
=.04\end{array}$ \\
\hline 2004 & Corral y Pinheiro & $\begin{array}{l}233 \text { estudiantes } \\
\text { de Hermosillo, } \\
\text { Sonora, México }\end{array}$ & $\begin{array}{l}\text { Establecieron que la efectividad (reacción } \\
\text { eficaz en el cuidado del medio ambiente), } \\
\text { la deliberación (estrategia para el bienestar } \\
\text { social, individual y de los organismos), la } \\
\text { anticipación (plan contingente que se aplicará } \\
\text { inmediatamente o a futuro), la solidaridad } \\
\text { (reacción altruista hacia los seres humanos, } \\
\text { especies animales y vegetales) y la austeridad } \\
\text { (forma de transformación y consumo mínimo } \\
\text { de los recursos naturales) son indicadores ( } \mathrm{R}^{2}= \\
.66, .69, .43, .33, .58, .29 \text { respectivamente) de la } \\
\text { conducta sustentable. }\end{array}$ & $\begin{array}{l}\mathrm{X}^{2}=14.4 ; 9 \mathrm{gl} ; \mathrm{p} \\
=.10 \mathrm{NNFI}=.95 \\
\mathrm{CFI}=.97, \mathrm{RMSEA} \\
=.05\end{array}$ \\
\hline 2004 & $\begin{array}{l}\text { Corral, Fraijó y } \\
\text { Tapia }\end{array}$ & $\begin{array}{l}95 \text { estudiantes de } \\
\text { México }\end{array}$ & $\begin{array}{l}\text { Demostraron la validez de los instrumentos } \\
\text { que miden siete factores psicoambientales; } \\
\text { creencias ecocéntricas }\left(\mathrm{R}_{1}=.70, \mathrm{R}_{2}=.59, \mathrm{R}_{3}\right. \\
\left.=.58, \mathrm{R}_{4}=.62, \mathrm{R}_{5}=.67 \text { y } \mathrm{R}_{6}=.61\right) \text {, creencias } \\
\text { antropocéntricas }\left(\mathrm{R}_{7}=.30, \mathrm{R}_{8}=.82, \mathrm{R}_{9}=.79 y\right. \\
\left.\mathrm{R}_{10}=.49\right) \text {, habilidades }\left(\mathrm{R}_{11}=.82, \mathrm{R}_{12}=.80, \mathrm{R}_{13}=\right. \\
\left..77, \mathrm{R}_{14}=.76 \text { y } \mathrm{R}_{15}=.56\right) \text {, motivos }\left(\mathrm{R}_{16}=.74, \mathrm{R}_{17}\right. \\
\left.=.87, \mathrm{R}_{18}=.85 \text { y } \mathrm{R}_{19}=.76\right) \text {, valores }\left(\mathrm{R}_{20}=.76, \mathrm{R}_{21}\right. \\
\left.=.90 \text { y } \mathrm{R}_{22}=.84\right) \text {, conocimientos }\left(\mathrm{R}_{23}=.49, \mathrm{R}_{24}\right. \\
=.37, \mathrm{R}_{25}=.47, \mathrm{R}_{26}=.40, \mathrm{R}_{27}=.25, \mathrm{R}_{28}=.31 \text { y } \\
\left.\mathrm{R}_{29}=.32\right) \text { y acciones }\left(\mathrm{R}_{30}=.63 \text { y } \mathrm{R}_{31}=.42\right) .\end{array}$ & $\begin{array}{l}\mathrm{X}^{2}=642.6 ; 433 \mathrm{gl} ; \\
\mathrm{p}<.001 \mathrm{BNNFI} \\
=.90 ; \mathrm{CFI}=.91 ; \\
\text { RMSEA }=.04\end{array}$ \\
\hline 2004 & Martínez & $\begin{array}{l}179 \text { residentes de } \\
\text { México }\end{array}$ & $\begin{array}{l}\text { Estableció la validez de tres instrumentos que } \\
\text { miden las variables preceptúales de juicios o } \\
\text { adscripciones de responsabilidad }\left(\mathrm{R}_{1}=.58, \mathrm{R}_{2}=\right. \\
\left..72, \mathrm{R}_{3}=.55, \mathrm{R}_{4}=.66 \text { y } \mathrm{R}_{5}=.61\right) \text {, responsabilidad } \\
\text { moral }\left(\mathrm{R}_{6}=.60, \mathrm{R}_{7}=.61, \mathrm{R}_{8}=.52, \mathrm{R}_{9}=.54, \mathrm{R}_{10}\right. \\
=.58) \text { y responsabilidad convencional }\left(\mathrm{R}_{11}=.63\right. \\
\left.\text { y } \mathrm{R}_{12}=.63\right) .\end{array}$ & $\begin{array}{l}\mathrm{X}^{2}=47-09 ; 51 \mathrm{gl} ; \\
\mathrm{p}<.001 \mathrm{GFI}=.95 ; \\
\text { AGFI }=.93 ; \mathrm{CFI}= \\
1: \mathrm{TU}=1 ; \mathrm{RMSEA} \\
=.00\end{array}$ \\
\hline
\end{tabular}


TABLA 3 (CONTINUACIÓN)

ESTRUCTURAS DE VALIDEZ

\begin{tabular}{|c|c|c|c|c|}
\hline AÑO & AUTOR & MUESTRA & ESTIMACIÓN & AJUSTE \\
\hline 2004 & $\begin{array}{l}\text { Valenzuela, } \\
\text { Corral, Quijada, } \\
\text { Griego, Ocaña y } \\
\text { Contreras }\end{array}$ & $\begin{array}{l}240 \text { residentes de } \\
\text { México }\end{array}$ & $\begin{array}{l}\text { Demostraron la validez de los instrumentos } \\
\text { que miden cuatro variables psicoambientales; } \\
\text { propensión al futuro (pesos factoriales de } \mathrm{R}_{1}= \\
.48, \mathrm{R}_{2}=.63, \mathrm{R}_{3}=.70, \mathrm{R}_{4}=.74, \mathrm{R}_{5}=.63, \mathrm{R}_{6}= \\
\left..66, \mathrm{R}_{7}=.70, \mathrm{R}_{8}=.40, \mathrm{R}_{9}=.63, \mathrm{R}_{10}=.67\right) \text {, auto- } \\
\text { reporte de ahorro de agua }\left(\mathrm{R}_{11}=.40, \mathrm{R}_{12}=.64\right. \\
\left.\mathrm{R}_{13}=.60, \mathrm{R}_{14}=.66\right) \text {, austeridad }\left(\mathrm{R}_{15}=.40, \mathrm{R}_{16}=\right. \\
.48, \mathrm{R}_{17}=.37, \mathrm{R}_{18}=.49, \mathrm{R}_{19}=.39, \mathrm{R}_{20}=.22 \text { y } \mathrm{R}_{21} \\
=.65) \text { y altruismo }\left(\mathrm{R}_{22}=.80, \mathrm{R}_{23}=.73, \mathrm{R}_{24}=.79\right. \\
\left.y \mathrm{R}_{25}=.78\right) .\end{array}$ & $\begin{array}{l}\mathrm{X}^{2}=430.6 ; 271 \\
\text { gl; } \mathrm{p}=.001 \mathrm{NNFI} \\
=.90 ; \mathrm{CFI}=.90 ; \\
\text { RMSEA }=.05\end{array}$ \\
\hline 2004 & Zúñiga y Asún & $\begin{array}{l}264 \text { residentes de } \\
\text { Chile }\end{array}$ & $\begin{array}{l}\text { Establecieron la validez del instrumento } \\
\text { que mide la identidad regional a partir de } \\
\text { tres factores; significado de pertenencia al } \\
\text { territorio }(.91) \text {, conciencia de pertenencia }(.72) \\
\text { y significado de pertenencia histórica cultural } \\
(.82) .\end{array}$ & $\begin{array}{l}\mathrm{X}^{2}=273 ; \mathrm{p}=.001 ; \\
\mathrm{CFI}=.97 \mathrm{NFI}= \\
.96 ; \mathrm{RMSEA}=.10\end{array}$ \\
\hline
\end{tabular}

Fuente: Elaboración propia.

De este modo, las estructuras de validez definirían a la sustentabilidad hídrica como un sistema de tarifas en función de la convergencia de rasgos cognitivos y comportamentales ante las contingencias de disponibilidad hídrica per cápita.

En este sentido:

$\diamond \quad$ El costo unitario del agua se incrementaría cuando los índices muestren un desajuste de las estructuras de validez. Este es el caso de individuos que no reflejan en sus respuestas la convergencia de síntomas de ahorro o dispendio. $\mathrm{O}$ bien, los pesos factoriales entre sus rasgos psicológicos son espurios de tal modo que la validez no puede ser demostrada. Es el caso de personas que creen intermitentemente en el equilibrio entre la disponibilidad de recursos y las necesidades humanas. En algunos casos, consideran que el crecimiento económico en detri- mento de la naturaleza es un derecho de la humanidad. En otros casos, consideran que la humanidad debería volver a una vida austera para construir un crecimiento económico a partir de energías abundantes y renovables.

$\diamond \quad$ El precio por unidad de agua disminuiría a partir de un ajuste estructural de la convergencia de sus rasgos psicológicos. Este es el caso de personas que tienden a creer en la escasez de recursos, valoran la distribución equitativa, perciben incertidumbre ante el desabasto, cuidan las instalaciones, se abastecen sin acaparamientos y reutilizan sus aguas jabonosas. Todos estos síntomas tendrían que converger en uno o más factores con pesos factoriales superiores a .300

$\diamond \quad$ La tarifa del servicio aumentaría en función del desajuste estructural de sus sín- 
tomas de ahorro o dispendio. Este es caso de usuarios que ahorran agua para sanear sus gastos, pero en el momento en que su ingreso aumente, tienden al dispendio. Se trata de rasgos psicológicos-conductuales en los que parecen converger en diversos factores o no alcanzan los pesos factoriales requeridos.

De este modo, la recaudación gubernamental no tendría que sancionar, incentivar o subsidiar el costo del servicio de agua potable al tener un diagnóstico exacto de las cogniciones $y$ acciones de los usuarios. Sin embargo, las estructuras de validez solo muestran la convergencia de síntomas en factores, por consiguien- te, la asociación entre factores permitiría un diagnóstico más preciso que las decisiones de consumo.

\section{ASOCIACIONES CAUSALES DE ACCIONES ANTI Y/O PRO SUSTENTABLES}

En este sentido, los factores más interrelacionados son antropocentrismo, ecocentrismo, frugalismo, altruismo, hedonismo, fatalismo y utilitarismo. Los índices de ajuste de estas estructuras demuestran la verosimilitud de suposiciones en torno al incremento o disminución de consumo unitario de agua. A partir de estos parámetros, se podrían establecer criterios tarifarios para el servicio de agua (ver tabla 4).

TABLA 4

ESTRUCTURAS DE COVARIANZAS

\begin{tabular}{|c|c|c|c|c|}
\hline AÑO & AUTOR & MUESTRA & ESTIMACIÓN & AJUSTE \\
\hline 2002 & Corral & $\begin{array}{l}195 \text { residentes de } \\
\text { México }\end{array}$ & $\begin{array}{l}\text { Demostró la validez discriminante entre el } \\
\text { paradigma de la excepcionalidad humana y nuevo } \\
\text { paradigma ambiental }(\mathrm{f}=.39) \text {. }\end{array}$ & $\begin{array}{l}\mathrm{X}^{2}=1.61 ; 8 \mathrm{gl} ; \mathrm{p}= \\
.99 ; \mathrm{NFI}=.99 ; \mathrm{NNFI} \\
=1.08 ; \mathrm{CFI}=1.00\end{array}$ \\
\hline 2004 & $\begin{array}{l}\text { Corral, Fraijó y } \\
\text { Tapia }\end{array}$ & $\begin{array}{l}95 \text { estudiantes de } \\
\text { México }\end{array}$ & $\begin{array}{l}\text { Demostraron la relación phi entre las siete } \\
\text { variables psicoambientales; conocimientos con } \\
\text { ecocentrismo }(\varphi=.74) \text {, con antropocentrismo } \\
\text { ( } \varphi=-.53) \text {, habilidades }(\varphi=.53) \text {, con acciones } \\
(\varphi=.46) \text { y con motivos }(\varphi=.37) \text {. Las creencias } \\
\text { ecocéntricas con los valores }(\varphi=-.69) \text { y con } \\
\text { las creencias antropocéntricas }(\varphi=-.73) \text {. Las } \\
\text { habilidades con el antropocentrismo }(\varphi=-.50) \text { y } \\
\text { con los valores }(\varphi=-.40) \text {. }\end{array}$ & $\begin{array}{l}\mathrm{X}^{2}=642.6 ; 433 \\
\text { gl; } \mathrm{p}<.001 ; \mathrm{BNNFI} \\
=.90 ; \mathrm{CFI}=.91 \\
\text { RMSEA }=.04\end{array}$ \\
\hline
\end{tabular}

Continúa... 
TABLA 4 (CONTINUACIÓN)

ESTRUCTURAS DE COVARIANZAS

\begin{tabular}{|c|c|c|c|c|}
\hline AÑO & AUTOR & MUESTRA & ESTIMACIÓN & AJUSTE \\
\hline 2004 & $\begin{array}{l}\text { Bustos, Flores, } \\
\text { Barrientos y } \\
\text { Martínez }\end{array}$ & $\begin{array}{l}210 \text { residentes de } \\
\text { México }\end{array}$ & $\begin{array}{l}\text { Demostraron que tanto el locus de control } \\
\text { Interno como los motivos de ahorro tuvieron una } \\
\text { correlación phi positiva y significativa }(\varphi=.49) \text {. }\end{array}$ & $\begin{array}{l}\text { Modelo } 1: \mathrm{X}^{2}= \\
335.7 ; 16 \text { gl; } \mathrm{p}< \\
.001 \text { Modelo 1: NFI } \\
=.93 \text { Modelo 2: NFI } \\
=.98 \\
\text { Modelo } 2: \mathrm{X}^{2}=78.7 ; \\
7 \mathrm{gl} ; \mathrm{p}<.001 ;\end{array}$ \\
\hline 2004 & $\begin{array}{l}\text { Corral, } \\
\text { Fraijó, Frías, } \\
\text { González y } \\
\text { Pinheiro }\end{array}$ & $\begin{array}{l}300 \text { residentes } \\
\text { de Hermosillo, } \\
\text { Sonora, México }\end{array}$ & $\begin{array}{l}\text { Establecieron que el presente tanto hedonista } \\
\text { como fatalista, covarían negativamente ( } \varphi= \\
\text {-.18; } \varphi=-.35 \text { ) con el ahorro de agua. Asimismo, } \\
\text { demostraron que la propensión al futuro también } \\
\text { tiene una estrecha relación positiva }(\varphi=.17) \text { con } \\
\text { la variable endógena de primer orden. A su vez, la } \\
\text { propensión al futuro tuvo una relación phi con el } \\
\text { pasado positivo ( } \varphi=.67) \text {, con el presente fatalista } \\
\text { ( } \varphi=.28) \text { y con el presente hedonista ( } \varphi=-.28) \text {. El } \\
\text { pasado negativo con el pasado positivo ( } \varphi=.26) \text {, } \\
\text { con el presente fatalista ( } \varphi=.44) \text { y con el presente } \\
\text { hedonista ( } \varphi=.21) \text {. El presente fatalista con el } \\
\text { presente hedonista }(\varphi=.65) \text {. }\end{array}$ & $\begin{array}{l}\mathrm{x}^{2}=351 ; 231 \mathrm{gl} ; \mathrm{p}< \\
.001 ; \mathrm{NNFI}=.90 ; \mathrm{CFI} \\
=.91 ; \mathrm{RMSEA}=.04\end{array}$ \\
\hline 2004 & $\begin{array}{l}\text { Corral y } \\
\text { Pinheiro }\end{array}$ & $\begin{array}{l}233 \text { estudiantes } \\
\text { de Hermosillo, } \\
\text { Sonora, México }\end{array}$ & $\begin{array}{l}\text { Establecieron las covarianzas directas, positivas } \\
\text { y significativas entre la anticipación con la } \\
\text { austeridad ( } \varphi=.48) \text {, con el altruismo ( } \varphi=.43) \text {, } \\
\text { con la efectividad ( } \varphi=.23 \text { ), con la deliberación ( } \varphi \\
=.16 \text { ) y con el ahorro de agua informado }(\varphi=.21) \text {. } \\
\text { Esta última variable con la austeridad ( } \varphi=.18) \text {, } \\
\text { con la deliberación ( } \varphi=.21 \text { ) y con la efectividad ( } \varphi \\
=.23 \text { ), se relacionó con la deliberación ( } \varphi=.22) \\
\text { y con el altruismo ( } \varphi=.25) \text {, el cual a su vez se } \\
\text { relacionó con la austeridad ( } \varphi=.36 \text { ) y finalmente, } \\
\text { con la deliberación }(\varphi=.16) \text {. }\end{array}$ & $\begin{array}{l}\mathrm{X}^{2}=14.4 ; 9 \mathrm{gl} ; \mathrm{p}= \\
.10 ; \mathrm{NNFI}=.95, \mathrm{CFI} \\
=.97, \mathrm{RMSEA}=.05\end{array}$ \\
\hline 2004 & Rego y Pereira & $\begin{array}{l}1201 \text { estudiantes } \\
\text { de Portugal }\end{array}$ & $\begin{array}{l}\text { Demostraron la relación phi entre el } \\
\text { comportamiento participativo con orientación } \\
\text { practica }(\varphi=.59) \text {. }\end{array}$ & $\begin{array}{l}\mathrm{X}^{2}=3.3 ; \text { RMSEA }= \\
.04 ; \mathrm{GFI}=.97 ; \mathrm{AGFI} \\
=.96 ; \mathrm{IFI}=.97 ; \mathrm{RFI} \\
=.97\end{array}$ \\
\hline 2004 & $\begin{array}{l}\text { Milfont y } \\
\text { Duckitt }\end{array}$ & $\begin{array}{l}455 \text { residentes de } \\
\text { Nueva Zelanda }\end{array}$ & $\begin{array}{l}\text { Establecieron la relación negativa entre el factor } \\
\text { conservacionista y el factor utilitarista }(\varphi=-.72) \text {. }\end{array}$ & $\begin{array}{l}\mathrm{X}^{2}=12.68 ; 29 \mathrm{gl} ; \\
\mathrm{p}=4.24 ; \mathrm{RMSEA}= \\
.08 ; \mathrm{SRMR}=.07 ; \mathrm{CFI} \\
=.91\end{array}$ \\
\hline
\end{tabular}


TABLA 4 (CONTINUACIÓN)

ESTRUCTURAS DE COVARIANZAS

\begin{tabular}{|c|c|c|c|c|}
\hline AÑO & AUTOR & MUESTRA & ESTIMACIÓN & AJUSTE \\
\hline 2006 & $\begin{array}{l}\text { Corral, Frías, } \\
\text { Fraijó y Tapia }\end{array}$ & $\begin{array}{l}150 \text { estudiantes } \\
\text { de Hermosillo, } \\
\text { Sonora, México }\end{array}$ & $\begin{array}{l}\text { Demostraron dos covarianzas: directas, negativas } \\
\text { y significativas entre el comportamiento } \\
\text { proambiental y su relación tanto con la } \\
\text { tendencia al riesgo ( } \varphi=-.12) \text { como con la falta } \\
\text { de autocontrol ( } \varphi=-.19) \text {. En contraste, dos } \\
\text { correlaciones: directas, positivas y significativas } \\
\text { entre la conducta antiambiental en relación } \\
\text { a la tendencia al riesgo ( } \varphi=.51) \text { y la falta de } \\
\text { autocontrol ( } \varphi=.27) \text {. Dos correlaciones: directas, } \\
\text { positivas y significativas entre la conducta } \\
\text { antisocial en relación con la tendencia al riesgo } \\
\text { ( } \varphi=.68) \text { y la falta de autocontrol ( } \varphi=.45 \text { ). } \\
\text { Finalmente, la correlación entre la conducta } \\
\text { antiambiental y la conducta antisocial resulta } \\
\text { positiva y significativa ( } \varphi=.84 \text { ) mientras la } \\
\text { correlación entre la tendencia al riesgo y la falta } \\
\text { de autocontrol resultaba también positiva y } \\
\text { significativa ( } \varphi=.48 \text { ). }\end{array}$ & $\begin{array}{l}\text { No presenta } \mathrm{X}^{2} ; \\
\text { no hay grados de } \\
\text { libertad y se reporta } \\
\text { una } \mathrm{p}<01 \text { BNNFI } \\
=.90 ; \mathrm{CFI}=.91 ; \\
\text { RMSEA }=.00\end{array}$ \\
\hline 2006 & $\begin{array}{l}\text { Bechtel, } \\
\text { Asai, Corral y } \\
\text { González }\end{array}$ & $\begin{array}{l}1358 \text { residentes } \\
\text { japoneses, } \\
\text { mexicanos, } \\
\text { peruanos y } \\
\text { estadounidenses }\end{array}$ & $\begin{array}{l}\text { Establecieron la estructura trifactorial (limites } \\
\text { al crecimiento, balance natural y excepción } \\
\text { humana) de la creencias ambientales. En el } \\
\text { primer, segundo y tercer modelo, el balance } \\
\text { natural tiene una covarianza positiva ( } \varphi=.41 ; \varphi= \\
.42 ; \varphi=.84 \text { ) con el factor de limite al crecimiento } \\
\text { respectivamente. }\end{array}$ & $\begin{array}{l}\text { Japón: } \mathrm{X}^{2}=64.6 ; 49 \\
\text { gl; } \mathrm{p}=.07 \mathrm{NNFI}= \\
.95 ; \mathrm{CFI}=.96 \\
\text { México: } \mathrm{X}^{2}=68.2 ; \\
48 \mathrm{gl} ; \mathrm{p}=.03 \mathrm{NNFI} \\
=.91 ; \mathrm{CFI}=.93 \\
\text { Perú: } \mathrm{X}^{2}=38.6 ; 50 \\
\text { gl; } \mathrm{p}=.89 ; \mathrm{NNFI}= \\
1 ; \mathrm{CFI}=1 \mathrm{EUA}: \mathrm{X}^{2} \\
=60.5 ; 49 \text { gl; } \mathrm{p}= \\
.12 \mathrm{NNFI}=.96 ; \mathrm{CFI} \\
=.97\end{array}$ \\
\hline 2006 & Corral y Frías & $\begin{array}{l}177 \text { residentes de } \\
\text { Sonora, México }\end{array}$ & $\begin{array}{l}\text { Establecieron la covariación entre las creencias } \\
\text { normativas y la conducta antisocial ( } \varphi=-.14) \text {. }\end{array}$ & $\begin{array}{l}\mathrm{X}^{2}=285.5 ; 203 \mathrm{gl} ; \\
\mathrm{p}<.001 ; \mathrm{NNFI}=.90 ; \\
\text { CFI }=.91 ; \mathrm{RMSEA} \\
=.000\end{array}$ \\
\hline
\end{tabular}

Fuente: Elaboración propia. 
Antes bien, es necesario advertir que los factores esgrimidos pertenecen a las creencias generales de escasez o abundancia de agua; es decir, se sabe que la interrelación entre el hedonismo, el fatalismo y el utilitarismo determinarían un alto consumo de agua. No obstante, el ecocentrismo, el frugalismo y el altruismo no garantizan un bajo consumo hídrico.

En consecuencia, la sustentabilidad hídrica sería un sistema de precios en función de las covariaciones entre los factores exógenos ante las contingencias de disponibilidad hídrica per cápita.

$\diamond \quad$ La cotización de cada unidad hídrica se incrementaría en función de un ajuste de la estructura covariable entre hedonismo, fatalismo y utilitarismo con asociaciones phi significativas, así como un desajuste en la estructura de covariación frugalista, ecocentrista $y$ altruista con niveles de covariación menores a .05 de significancia. Es el caso de usuarios que creen en el fin inmediato del mundo y tienden a consumir indiscriminadamente. Se trata también de usuarios que tienden a creer que la disponibilidad hídrica global no puede ser determinada por sus actos.

El precio unitario de agua disminuiría a partir de un ajuste de la estructura de covarianza del altruismo, frugalismo $y$ ecocentrismo. Es el caso de individuos que tienden a preservar el líquido al creer que una vida austera es mejor que una consumista, que ayudar a la gente es mejor que competir con ella y que la naturaleza es mejor que la humanidad. También el costo disminuiría si la estructura de covarianzas del hedonismo, fatalismo y utilitarismo presentaran un desajuste. Esto supondría que las personas abandonan las creencias de abundancia de recursos, exclusividad humana $y$ fin inmediato de la naturaleza.

$\diamond \quad$ La tarifa de cada unidad de agua aumentaría si la estructura de covarianza tuviera un ajuste para las seis creencias con covarianzas phi con niveles de significancia insuficientes a los requeridos. Este sería el caso de personas que están convencidas del fin de mundo pero creen en la preservación del patrimonio para sus hijos y en la competencia por los recursos, así como, creen en la ayuda a sus familiares, en el disfrute aquí y ahora de la vida, al mismo tiempo, en la utilización de la naturaleza para tal propósito.

Las estructuras de covariación permiten establecer un sistema diferencial de tarifas pero no establecen predicciones a futuro. Las estructuras de predicción serían más idóneas al momento de establecer tarifas prospectivas.

Baron y Kenny (1996) demostraron que la covarianza entre dos factores exógenos determina la variabilidad de un factor endógeno. A este efecto le llamaron "moderación". Se trata de la predicción de un comportamiento a partir de la interacción de dos creencias diferentes. Es el caso de individuos con altos ingresos que creen en la prosperidad pero al mismo tiempo creen en un mejor futuro ambiental para sus hijos y por ello ahorran recursos.

En este sentido, un comportamiento proambiental podría estar determinado indirectamente por creencias generales e influido directamente, por actitudes específicas. Este efecto se conoce como "mediación". Se trata de un modelo en el que la covariación entre creencias incide en el comportamiento a través de actitudes o motivos.

A continuación se exponen ambos efectos en estructuras de predicción.

\section{PREDICCIONES CAUSALES DE ACCIONES ANTI Y/O PRO SUSTENTABLES}

Las estructuras de predicción demuestran la relación causal entre factores de creencias, actitudes y motivos sobre los comportamientos a favor o en contra del medio ambiente (ver tabla 5).

Se trata de modelos estructurales con factores exógenos de creencias de escasez y abundancia de agua que inciden indirectamente en factores comportamentales de ahorro $y$ dispendio. Dicho efecto, esta mediado por factores endógenos de actitudes y motivos. 
TABLA 5

ESTRUCTURAS DE PREDICCIONES

\begin{tabular}{|c|c|c|c|c|}
\hline AÑO & AUTOR & MUESTRA & ESTIMACIÓN & AJUSTE \\
\hline 1995 & Grob & $\begin{array}{l}398 \text { residentes de } \\
\text { Canton Berne, } \\
\text { Suiza }\end{array}$ & $\begin{array}{l}\text { Estableció que el componente emocional de } \\
\text { la actitud predice la conducta proambiental } \\
(\beta=.20) \text {. }\end{array}$ & $\begin{array}{l}\mathrm{X}^{2}=8.87 ; 7 \mathrm{gl} ; \mathrm{p}=.26 \\
\mathrm{AGFI}=.99 ; \mathrm{RMSR}=.01\end{array}$ \\
\hline 1996 & Obregón & $\begin{array}{l}100 \text { familias de } \\
\text { Sonora, México }\end{array}$ & $\begin{array}{l}\text { Demostró que las creencias de } \\
\text { conservación determinan la reutilización } \\
\text { observada }(\beta=.23) \text { y el autorreporte } \\
\text { de reciclaje }(\beta=.241) \text {. Por su parte, las } \\
\text { creencias de austeridad inciden sobre } \\
\text { la reutilización reportada ( } \beta=.20) \text { y } \\
\text { finalmente, las creencias materiales } \\
\text { predicen el autorreporte de reciclaje }(\beta= \\
.329 \text {, y el reciclaje observado }(\beta=.227) \text {. }\end{array}$ & $\begin{array}{l}\text { Reutilización con } \mathrm{X}^{2}= \\
64.46 ; \mathrm{p}=.002 \mathrm{IAC}=.99 \\
\text { Reciclaje con } \mathrm{X}^{2}= \\
56.27 ; \mathrm{p}=.016 \mathrm{IAC}=.94\end{array}$ \\
\hline 1997 & Corral & $\begin{array}{l}100 \text { residentes } \\
\text { de Hermosillo, } \\
\text { Sonora, México }\end{array}$ & $\begin{array}{l}\text { Estableció que las creencias predicen } \\
\text { significativamente el reuso y } \\
\text { reciclaje reportado ( } \beta=.25 \text { y } \beta=.31 \text {, } \\
\text { respectivamente). Además, los motivos } \\
\text { determinan significativamente el reuso } \\
\text { y el reciclaje observado ( } \beta=.47 \text { y .31, } \\
\text { respectivamente). }\end{array}$ & $\begin{array}{l}\text { Reutilización: } \mathrm{X}^{2}= \\
\text { 34.78: } 29 \text { gl: } \mathrm{p}=.21 \mathrm{CFI} \\
=.90 \\
\text { Reciclaje: } \mathrm{X}^{2}=26.71 ; 29 \\
\mathrm{gl} ; \mathrm{p}=.58 \mathrm{CFI}=1\end{array}$ \\
\hline 2001 & $\begin{array}{l}\text { Corral, Frías y } \\
\text { González }\end{array}$ & $\begin{array}{l}114 \text { residentes de } \\
\text { Sonora, México }\end{array}$ & $\begin{array}{l}\text { Demostraron el efecto directo, positivo } \\
y \text { significativo entre el comportamiento } \\
\text { antisocial sobre el desperdicio de agua ( } \beta \\
=.35 \text { ). }\end{array}$ & $\begin{array}{l}\mathrm{X}^{2}=26,25 \mathrm{gl} ; \mathrm{p}=.36 \\
\mathrm{NNFI}=.95, \mathrm{CFI}=.96 \\
\text { RMSEA }=.02\end{array}$ \\
\hline 2002 & $\begin{array}{l}\text { Espinosa, Orduña } \\
y \text { Corral }\end{array}$ & $\begin{array}{l}485 \text { residentes } \\
\text { de Hermosillo, } \\
\text { Sonora, México }\end{array}$ & $\begin{array}{l}\text { Establecieron la predicción de las } \\
\text { competencias sobre el ahorro de agua ( } \beta \\
=.32) \text {. }\end{array}$ & $\begin{array}{l}\mathrm{X}^{2}=271.5 ; 84 \mathrm{gl} ; \mathrm{p}< \\
.001 ; \mathrm{NFI}=.90 ; \mathrm{CFI}= \\
.93 ; \text { RMSEA }=.03\end{array}$ \\
\hline 2002 & Corral & $\begin{array}{l}195 \text { residentes de } \\
\text { Sonora, México }\end{array}$ & $\begin{array}{l}\text { Estableció el efecto indirecto, positivo } \\
\text { y significativo del nuevo paradigma } \\
\text { ambiental sobre el reuso }(\beta=.24) \text {. } \\
\text { Estableció la determinación indirecta } \\
\text { y negativa del paradigma de la } \\
\text { excepcionalidad humana sobre el reuso ( } \beta \\
=-.19 \text { ). Ambos efectos estuvieron mediados } \\
\text { por los motivos. }\end{array}$ & $\begin{array}{l}\mathrm{X}^{2}=91.42 ; 73 \mathrm{gl} ; \mathrm{p}= \\
.07 ; \mathrm{NFI}=.87 ; \mathrm{NNFI}= \\
.94 ; \mathrm{CFI}=.93\end{array}$ \\
\hline
\end{tabular}

Continúa... 
TABLA 5 (CONTINUACIÓN)

ESTRUCTURAS DE PREDICCIONES

\begin{tabular}{|c|c|c|c|c|}
\hline AÑO & AUTOR & MUESTRA & ESTIMACIÓN & AJUSTE \\
\hline 2002 & Corral & $\begin{array}{l}195 \text { residentes } \\
\text { de Hermosillo, } \\
\text { Sonora, México }\end{array}$ & $\begin{array}{l}\text { Demostró que los motivos para reutilizar } \\
\text { ropa, cajas, vidrio y periódico son } \\
\text { predictores de la reutilización ( } \beta=.64) \text {. }\end{array}$ & $\begin{array}{l}\mathrm{X}^{2}=249,7 ; 103 \mathrm{gl} ; \mathrm{p}< \\
.001 ; \text { IANN }=0,91 ; \mathrm{IAC}= \\
0,93 ; \text { GFI }=1,0 ; \text { AGFI }= \\
.91 ; \text { RQQMEA }=0,04\end{array}$ \\
\hline 2003 & $\begin{array}{l}\text { Corral, Frías y } \\
\text { González }\end{array}$ & $\begin{array}{l}114 \text { residentes de } \\
\text { Sonora, México }\end{array}$ & $\begin{array}{l}\text { Demostraron el efecto directo y positivo } \\
(\beta=.35) \text { de la conducta antisocial sobre el } \\
\text { dispendio hidrológico. }\end{array}$ & $\begin{array}{l}\mathrm{X}^{2}=26 ; 25 \mathrm{gl} ; \mathrm{p}>.05 \\
\text { NNFI }=.95 ; \text { CFI }=.96 \\
\text { RMSEA }=.02\end{array}$ \\
\hline 2003 & $\begin{array}{l}\text { Corral, Frías y } \\
\text { González }\end{array}$ & $\begin{array}{l}114 \text { residentes de } \\
\text { Sonora, México }\end{array}$ & $\begin{array}{l}\text { Establecieron el efecto directo y positivo de } \\
\text { la conducta antisocial sobre el dispendio } \\
\text { hidrológico }(\beta=.37) \text {. }\end{array}$ & $\begin{array}{l}\mathrm{X}^{2}=26 ; 25 \mathrm{gl} ; \mathrm{p}>.05 \\
\mathrm{NNFI}=.95 ; \mathrm{CFI}=.96 \\
\text { RMSEA }=.02\end{array}$ \\
\hline 2004 & Milfont y Duckitt & $\begin{array}{l}455 \text { residentes de } \\
\text { Nueva Zelanda }\end{array}$ & $\begin{array}{l}\text { Establecieron el efecto directo del factor } \\
\text { conservacionista de segundo orden sobre la } \\
\text { conducta autoreportada ( } \beta=.67 \text { ). }\end{array}$ & $\begin{array}{l}\mathrm{X}^{2}=12.68 ; 29 \mathrm{gl} ; \mathrm{p}= \\
4.24 \text { RMSEA }=.08 ; \text { SRMR } \\
=.07 ; \text { CFI }=.91\end{array}$ \\
\hline 2004 & Bustos & $\begin{array}{l}399 \text { amas de casa } \\
\text { de México }\end{array}$ & $\begin{array}{l}\text { Demostró la incidencia de las creencias } \\
\text { de obligación de ahorrar agua sobre las } \\
\text { habilidades efectivas ( } \beta=.21 \text { ). A su vez, las } \\
\text { habilidades efectivas determinan ( } \beta=.31 \text { ) } \\
\text { el comportamiento pro ambiental (aseo } \\
\text { personal y la preparación de alimentos). } \\
\text { Estableció que el locus de control interno } \\
\text { incide directa y positivamente sobre las } \\
\text { creencias de obligación }(\beta=.37) \text {. }\end{array}$ & $\begin{array}{l}\mathrm{X}^{2}=17.17 ; 13 \mathrm{gl} ; \mathrm{p}>.05 ; \\
\mathrm{NNFI}=.99 ; \mathrm{RMSEA}=.03\end{array}$ \\
\hline 2004 & $\begin{array}{l}\text { Fraijo, Tapía y } \\
\text { Corral }\end{array}$ & $\begin{array}{l}118 \text { residentes de } \\
\text { Sonora, México }\end{array}$ & $\begin{array}{l}\text { Demostraron el efecto directo de una } \\
\text { intervención sobre la estructura de las } \\
\text { competencias de ahorro de agua ( } \beta= \\
.98 \text { ), la cual incluye como indicadores } \\
\text { a las creencias, las habilidades, los } \\
\text { conocimientos y los motivos en orden } \\
\text { de importancia. En consecuencia, las } \\
\text { competencias de ahorro de agua tuvieron } \\
\text { un efecto directo, negativo y significativo } \\
\text { ( } \beta=-.15 \text { ) sobre el consumo observado } \\
\text { y registrado de agua. Por lo tanto, el } \\
\text { programa de educación ambiental aplicado } \\
\text { en esta muestra contribuyó a un mejor } \\
\text { ahorro de agua vía las competencias. }\end{array}$ & $\begin{array}{l}\mathrm{X}^{2}=479.78 ; 294 \mathrm{gl} ; \mathrm{p}= \\
.001 \mathrm{NNFI}=.91 ; \mathrm{CFI}= \\
.92 ; \mathrm{RMSEA}=.06\end{array}$ \\
\hline
\end{tabular}

Continúa... 
TABLA 5 (CONTINUACIÓN)

ESTRUCTURAS DE PREDICCIONES

\begin{tabular}{|c|c|c|c|c|}
\hline AÑO & AUTOR & MUESTRA & ESTIMACIÓN & AJUSTE \\
\hline 2004 & $\begin{array}{l}\text { Corral, Quijada, } \\
\text { Griego, Ocaña y } \\
\text { Contreras }\end{array}$ & $\begin{array}{l}240 \text { residentes } \\
\text { de Hermosillo y } \\
\text { Ciudad Obregón, } \\
\text { Sonora, México }\end{array}$ & $\begin{array}{l}\text { Establecieron que la propensión al } \\
\text { futuro predice directa, positiva } y \\
\text { significativamente }(\beta=.40 ; \mathrm{p}<.05) \text { a la } \\
\text { austeridad misma que a su vez también es } \\
\text { predecida ( } \beta=.23 ; \mathrm{p}<.05) \text { por el altruismo } \\
\text { y predictora }(\beta=.37) \text { del ahorro de agua. }\end{array}$ & $\begin{array}{l}\mathrm{X}^{2}=430.6 ; 271 \mathrm{gl} ; \mathrm{p}= \\
.001 \mathrm{NNFI}=.90 ; \mathrm{CFI}= \\
.90 ; \text { RMSEA }=.05\end{array}$ \\
\hline 2004 & $\begin{array}{l}\text { Valenzuela, } \\
\text { Corral, Quijada, } \\
\text { Griego, Ocaña y } \\
\text { Contreras }\end{array}$ & $\begin{array}{l}240 \text { residentes de } \\
\text { México }\end{array}$ & $\begin{array}{l}\text { Demostraron que la propensión al } \\
\text { futuro predice directa, positiva y } \\
\text { significativamente ( } \beta=.40 \text { ) a la austeridad } \\
\text { misma que a su vez también es causada ( } \beta \\
=.23 \text { ) por el altruismo y a su vez incide ( } \beta \\
=.37 \text { ) el ahorro de agua explicando el } 14 \% \\
\text { de su varianza. }\end{array}$ & $\begin{array}{l}\mathrm{X}^{2}=430.6 ; 271 \mathrm{gl} ; \mathrm{p}= \\
.001 \mathrm{NNFI}=.90 ; \mathrm{CFI}= \\
.90 ; \text { RMSEA }=.05\end{array}$ \\
\hline 2004 & $\begin{array}{l}\text { Bustos, Flores, } \\
\text { Barrientos y } \\
\text { Martínez }\end{array}$ & $\begin{array}{l}210 \text { residentes de } \\
\text { México }\end{array}$ & $\begin{array}{l}\text { Demostraron que tanto el locus de control } \\
\text { interno ( } \beta=.44) \text { como los motivos de } \\
\text { ahorro ( } \beta=.67 \text { ) predicen directa, positiva } \\
\text { y significativamente el ahorro de agua en } \\
\text { el aseo personal explicando el } 64 \% \text { de la } \\
\text { varianza. En un segundo modelo, tanto el } \\
\text { locus de control interno ( } \beta=.23 \text { ) como los } \\
\text { motivos de ahorro ( } \beta=.29 \text { ) determinan la } \\
\text { limpieza general explicando el } 13 \% \text {. }\end{array}$ & $\begin{array}{l}\text { Modelo } 1: \mathrm{X}^{2}=335.7 ; 16 \\
\text { gl; } \mathrm{p}<.001: \mathrm{NFI}=.93 \\
\text { Modelo } 2: \mathrm{X}^{2}=78.7 ; 7 \\
\text { gl; } \mathrm{p}<.001 \mathrm{NFI}=.98\end{array}$ \\
\hline 2004 & $\begin{array}{l}\text { Bustos, Flores y } \\
\text { Andrade }\end{array}$ & $\begin{array}{l}349 \text { residentes de } \\
\text { México }\end{array}$ & $\begin{array}{l}\text { Establecieron el efecto directo, positivo y } \\
\text { significativo del locus de control interno } \\
\text { sobre el ahorro de agua ( } \beta=.14 \text { ) y el efecto } \\
\text { indirecto en tres senderos; el primero } \\
\text { a través de las creencias de obligación } \\
\text { de cuidar el agua ( } \beta=.43 \text { ), las cuales } \\
\text { determinan las habilidades instrumentales } \\
\text { ( } \beta=.20) \text { y estas, el ahorro de agua ( } \beta= \\
.36) \text {. La segunda trayectoria a través de } \\
\text { los motivos de protección socio-ambiental } \\
\text { ( } \beta=.21) \text { quienes influyen en el ahorro de } \\
\text { agua ( } \beta=.14 \text { ) y la tercera ruta a través de la } \\
\text { percepción de riesgo a la salud ( } \beta=.30 \text { ) que } \\
\text { causa los motivos de protección ambiental } \\
\text { socio-ambiental ( } \beta=.20 \text { ). Además, } \\
\text { establecieron el efecto indirecto de los } \\
\text { conocimientos a través de las habilidades } \\
\text { instrumentales ( } \beta=.07 \text { ). }\end{array}$ & $\begin{array}{l}\mathrm{X}^{2}=.35 ; 10 \mathrm{gl} ; \mathrm{p}=.000 ; \\
\mathrm{R}^{2}=.25 \mathrm{GFI}=.97 ; \text { AGFI } \\
=.92 ; \mathrm{RMSEA}=.08\end{array}$ \\
\hline
\end{tabular}




\begin{tabular}{|c|c|c|c|c|}
\hline AÑO & AUTOR & MUESTRA & ESTIMACIÓN & AJUSTE \\
\hline 2006 & Milfont $y$ Duckitt & $\begin{array}{l}314 \text { estudiantes de } \\
\text { Nueva Zelanda }\end{array}$ & $\begin{array}{l}\text { Demostraron el efecto directo, positivo } \\
\text { y significativo }(\beta=.71) \text { de la dimensión } \\
\text { (utilitaria) de las creencias y actitudes } \\
\text { ambientales sobre el comportamiento } \\
\text { proambiental. }\end{array}$ & $\begin{array}{l}\text { Primer modelo: } \mathrm{X}^{2}= \\
997.88 ; 582 \text { gl; RMSEA } \\
=.04 ; \mathrm{SRMR}=.06 ; \mathrm{CFI}= \\
.98 ; \mathrm{ECVL}=3.72 ; \mathrm{CALC}= \\
1564.83 \\
\text { Segundo modelo: } \mathrm{X}^{2}= \\
946.88 ; 581 \text { gl RMSEA = } \\
.04 ; \mathrm{SRMR}=.06 ; \mathrm{CFI}= \\
.98 ; \mathrm{ECVI}=3.57 ; \mathrm{CALC}= \\
1520.66\end{array}$ \\
\hline 2006 & Corral y Frías & $\begin{array}{l}177 \text { residentes de } \\
\text { Sonora, México }\end{array}$ & $\begin{array}{l}\text { Demostraron el efecto directo de las } \\
\text { creencias normativas y la conducta } \\
\text { antisocial ( } \beta=.22 \text { y -.18, respectivamente) } \\
\text { sobre la conservación de agua. }\end{array}$ & $\begin{array}{l}\mathrm{X}^{2}=285.5 ; 203 \mathrm{gl} ; \mathrm{p}< \\
.001 \mathrm{NNFI}=.90 ; \mathrm{CFI}= \\
.91 ; \text { RMSEA }=.000\end{array}$ \\
\hline 2006 & McFarie y Hunt & $\begin{array}{l}3052 \text { residentes de } \\
\text { Canadá }\end{array}$ & $\begin{array}{l}\text { Establecieron los efectos indirectos, } \\
\text { negativos y significativos de los valores } \\
\text { y los conocimientos sobre el activismo } \\
\text { ecológico ( } \beta=-.54 ; \beta=-.08 \text { ). Dicho efecto } \\
\text { esta mediado por las actitudes ( } \beta=-.25 \text { ). }\end{array}$ & $\begin{array}{l}\mathrm{X}^{2}=1.91 ; 2 \mathrm{gl} ; \mathrm{p}=.38 \\
\text { AGFI }=.99 ; \mathrm{RMSEA}= \\
.000\end{array}$ \\
\hline
\end{tabular}

Fuente: Elaboración propia.

Sin embargo, Ajzen (2001) ha cuestionado la relación entre una creencia global y un comportamiento específico. A decir de este autor, las estructuras de conductas específicas necesariamente se relacionan con estructuras de creencias específicas. Los estudios psicológicos de la sustentabilidad iniciaron con estructuras generales de creencias, tales como, el Paradigma Social Dominante, Paradigma de la Excepción y Humana y Nuevo Paradigma Ecológico, en las que sus indicadores también referían aseveraciones generales. Transcurridas dos décadas, se adoptaron escalas más específicas para establecer estructuras de convergencia. Este sería el caso de las estructuras de percepciones que se refieren a un cálculo de costos $y$ beneficios, a partir de las cuales se minimiza la incertidumbre y se maximiza la utilidad a través de decisiones de riesgo. También sería el caso de las estructuras de motivos que se refieren a beneficios económicos al momento de llevar a cabo una acción sustentable. O bien, es el caso de las estructuras de habilidades, las cuales se refieren a acciones específicas constantes como respuestas a las contingencias de escasez de recursos. En el caso de las estructuras de competencias, estas son sumamente puntuales puesto que se refieren a adaptaciones e innovaciones en función de variaciones de escasez o abundancia de recursos. Finalmente, la asociación de habilidades y competencias daría origen a comportamientos sustentables en los que las personas sistematizan deliberada y planificadamente, acciones a favor de la conservación de recursos.

En tal sentido, la sustentabilidad hídrica se refiere a un sistema de tarifas en función 
de las relaciones causales directas e indirectas de factores exógenos sobre factores endógenos ante las contingencias de disponibilidad hídrica per cápita. De este modo:

$\diamond \quad$ Los costos del servicio de agua se incrementarían cuando los valores gamma 0 beta no sean significativos y/o los índices de ajuste no se acerquen a la unidad $y / 0$ los residuales no se aproximen al cero. Es el caso de personas que creen en la escasez de recursos, pero también creen en el disfrute pleno de la vida y por ello, tienden al dispendio.

$\diamond \quad$ Las tarifas disminuirían siempre y cuando la interacción entre creencias influya en el ahorro de agua. Obviamente, los parámetros de predicción deberán ser significativos, los estadísticos de ajuste cercanos a la unidad y los índices residuales próximos al cero. Se estaría ante un escenario ideal en el que los usuarios creen en la escasez de recursos, forman actitudes afectivo-racionales, toman decisiones de minimización de costos $y$ maximización de beneficios que los llevan a ahorrar recursos.

$\diamond \quad$ Los precios del servicio aumentarían en función de desajustes en las estructuras de predicción sin importar la significancia de los parámetros de causalidad. Es el caso de usuarios, los cuales no puede establecer un modelo hipotético que se acerque a sus usos y costumbres concediendo que respondieron honestamente a las entrevistas.

Las estructuras de predicción solo muestran las relaciones causales entre factores incluidos en un modelo. En estos modelos: valores, creencias, percepciones, actitudes, identidades, motivos, conocimientos, habilidades, competencias e intenciones son determinantes del ahorro o dispendio de recursos. Hasta el momento no ha sido posible integrar dichas variables en una sola estructura y por ello, las estructuras de disturbio cobran relevancia porque muestran el efecto de variables excluidas de los modelos.

\section{ERRORES CAUSALES DE ACCIONES ANTI Y/O PRO SUSTENTABLES}

Las estructuras de disturbio o errores de medición son exiguos en los estudios psicológicos de la sustentabilidad (ver tabla 6).

El parámetro sigma $(\varsigma)$ muestra el efecto de variables no incluidas en las estructura. Si se considera que la explicación ideal de la variabilidad de un comportamiento es cercano a la unidad, un valor sigma aproximado a la unidad compromete la verosimilitud de las relaciones correlacionales y causales del modelo. En tal sentido, las estructuras de disturbio aspiran a reducir el valor sigma para explicar la mayor cantidad de varianza.

TABLA 6

ESTRUCTURAS DE DISTURBIOS

\begin{tabular}{|c|c|c|c|c|}
\hline AÑO & AUTOR & MUESTRA & ESTIMACIÓN & AJUSTE \\
\hline 2001 & $\begin{array}{l}\text { Corral y } \\
\text { Encinas }\end{array}$ & $\begin{array}{l}100 \text { amas de casa de } \\
\text { Hermosillo, Sonora, México }\end{array}$ & $\begin{array}{l}\text { Demostraron que los motivos } \\
\text { explican el reciclaje de papel } \\
\left(\mathrm{R}^{2}=.52 ; \varsigma=.48\right)\end{array}$ & $\begin{array}{l}\mathrm{X}^{2}=48.1 ; 42 \mathrm{gl} ; \mathrm{p}=.24 ; \text { IBANN } \\
=.88 ; \mathrm{IAC}=.92\end{array}$ \\
\hline 2002 & Corral & $\begin{array}{l}200 \text { residentes de Ciudad } \\
\text { Obregón, Sonora, México }\end{array}$ & $\begin{array}{l}\text { Estableció que las } \\
\text { competencias proambientales } \\
\text { explican el ahorro de agua }\left(\mathrm{R}^{2}\right. \\
=.54 ; \varsigma=.46)\end{array}$ & $\begin{array}{l}\mathrm{X}^{2}=43 ; 34 \mathrm{gl} ; \mathrm{p}=.47 ; \mathrm{NFI}= \\
.95 ; \mathrm{NNFI}=1 ; \mathrm{CFI}=1\end{array}$ \\
\hline 2003 & Corral & $\begin{array}{l}500 \text { residentes de Hermosillo } \\
\text { y Ciudad Obregón, Sonora, } \\
\text { México }\end{array}$ & $\begin{array}{l}\text { Estableció que el utilitarismo } \\
\text { explica la variabilidad del } \\
\text { consumo de agua }\left(\mathrm{R}^{2}=.22 ; \varsigma\right. \\
=.78) .\end{array}$ & $\begin{array}{l}\mathrm{X}^{2}=249,7 ; 103 \mathrm{gl} ; \mathrm{p}<.001 ; \\
\text { IANN = 0,91; IAC = .93; GFI = } 1 ; \\
\text { RQQMEA = .04 }\end{array}$ \\
\hline
\end{tabular}

Fuente: Elaboración propia 
Considerando el valor sigma, la sustentabilidad hídrica se definiría como un sistema de precios en función de la variabilidad explicada de factores exógenos y endógenos del modelo, así como, la variabilidad no explicada (disturbio) de factores excluidos del modelo ante las contingencias de disponibilidad hídrica per cápita. Las implicaciones de esta definición serían:

$\diamond \quad$ Un incremento de las tarifas se efectuaría a la par de un aumento en el disturbio de un modelo estructural. Este es el caso de personas que fueron entrevistadas o respondieron a un número inferior de reactivos psicológicos de los que debieran haber contestado.

$\diamond \quad$ Una disminución de los precios a partir de un valor sigma cercano al cero. Es el caso de personas que fueron encuestadas o respondieron a preguntas y reactivos que miden todas las variables psicológicas y comportamentales posibles.

$\diamond \quad$ Un aumento de los precios por la ausencia del parámetro sigma en el estudio. Este es el caso de modelos estructurales que ofrecen valores de disturbio y por ende, no es posible establecer el efecto de otras variables excluidas en el modelo.

Hasta aquí la revisión de estructuras cognitivo-conductuales que aportaría nuevos criterios de aumento o disminución de los precios unitarios del servicio de agua.

\section{CONCLUSIÓN}

A partir de los modelos esgrimidos, la sustentabilidad hídrica se definiría como un sistema de tarifas en función de la convergencia de rasgos cognitivos y comportamentales, de la covariación entre factores exógenos, de las relaciones causales directas e indirectas de factores exógenos sobre factores endógenos $y$ de la variabilidad explicada de factores exógenos y endógenos incluidos en el modelo, así como, la variabilidad no explicada (disturbio) de factores excluidos del modelo ante las contingencias de disponibilidad hídrica per cápita.
Es decir, la sustentabilidad hídrica estaría determinada por las contingencias de disponibilidad hídrica per cápita y su relación con rasgos y factores cognitivo-conductuales. Ambos, establecerían un sistema de precios acorde a los valores, creencias, percepciones, identidades, actitudes, motivos, conocimientos, habilidades, competencias, intenciones y comportamientos de los usuarios que derrochan o ahorran agua.

\section{REFERENCIAS}

Aitken, C. y McMahon, T. "Residential water use: predictive and reducing consumption". Journal of Applied Social Psychology 24. 1994: 136-158.

Ajzen, I. "Nature and operation of attitudes”. Annual Review Psychology 52. 2001: 27-58.

Auxiliadora, M. y Manera, J. "El análisis factorial por componentes principales". Análisis multivariable para las ciencias sociales. Levy, J. P. y Varela, J. (coords.). Madrid. Pearson Prentice Hall, 2003: 327-360.

Baron, R. y Kenny, D. "The moderator and mediator variables distinction in social psychology research. Conceptual, strategic, and statistical considerations". Journal of Personality and Social Psychology 51. 1986: 1173-1182.

Bechtel, R.; Asai, M.; Corral, V. y González, A. "A cross cultural study of environmental beliefs structures in USA, Japan, Mexico and Peru". International Journal of Psychology 41. 2006: 145-151.

Bechtel, R.; Corral, V. y Pinheiro, J. Q. "Environmental belief systems United Status, Brazil and Mexico". Journal of Cross-Cultural Psychology 30. 1999: 122-128.

Berk, R. A.; Cooley, T. F.; LaCivita, C. J.; Parker, S.; Sreld, K. y Brewer, M. B. "Reducing consumption in periods of acute scarcity: the case of water". Social Science Research 9. 1980: 99-120. 
Bernard, P. "Ecological implications of water spirit beliefs in Southern Africa: the need to protect knowledge, nature and resource rights". USDA Forest Service Proceeding RMRS-P-2. 2003: 148-153.

Bonaiuto, M.; Bilotta, E.; Bonnes, M.; Carrus, G.; Ceccarelli, M. y Martorella, H. "Local identity moderating the role of individual differences in natural resource use: the case of water consumption". Journal of Applied Social Psychology 38. 2008: 947-967.

Bridgeman, J. "Public perception towards water recycling in California". Water and Environment Journal 18. 2004: 150-154.

Bustos, J. "Modelo de conducta proambiental para el estudio de la conservación de agua potable". [Tesis de Doctorado] Facultad de Psicología de la Universidad Nacional Autónoma de México, 2004.

Bustos, J. y Flores, L. "Evaluación de problemas ambientales, calidad del ambiente y creencias de afectación de la salud". La Psicología Social en México 8. 2000: 445-451.

Bustos, J.; Flores, L. y Andrade, P. "Motivos $y$ percepción de riesgos como factores antecedentes a la conservación de agua en la Ciudad de México". La Psicología Social en México 9. 2002: 611-617.

Bustos, J.; Flores, L.; Barrientos, C. y Martínez, J. "Ayudando a contrarrestar el deterioro ecológico: atribución y motivos para conservar agua". La Psicología Social en México 10. 2004: 521-526.

Bustos, J.; Flores, M. y Andrade, P. "Predicción de la conservación de agua a partir de factores socio cognitivos". Medio Ambiente y Comportamiento Humano 5. 2004: 53-70.

Bustos, J.; Montero, M. y Flores, L. Tres diseños de intervención antecedente para promover. 2002.

Chang, H.; Parandvash, G. y Shandas, V. "Spatial variations of single-family residential water consumption in Portland, Oregon". Urban Geography 31 (7). 2010: 953-972.
Corral, V. "Interacciones ambiente/conducta: algunas áreas de investigación”. Las aportaciones mexicanas a la psicología. Alcaraz, V. M. y Bouzas, A. (coords.). México: UNAM, 1998: 55-70.

Corral, V. "La definición del comportamiento proambiental”. La Psicología Social en México 8. 2000: 466-472.

Corral, V. "Aplicaciones del Modelamiento Estructural a la investigación psicológica”. Revista Mexicana de Psicología 18. 2001: 193-209.

Corral, V. "A structural model of proenvironmental competency". Environment \& Behavior 34. 2002a: 531-549.

Corral, V. "Avances y limitaciones en la medición del comportamiento proambiental”. Temas Selectos de Psicología Ambiental. Guevara, J. y Mercado, S. (coords.). México: UNAMGRECO-UNILIBRE, 2002b: 483-510.

Corral, V. "Structural equation modelling". Handbook of Environmental Psychology. Bechtel, R. y Churcman, A. (eds.). New York. Wiley \& Sons Inc., 2002c: 256-270.

Corral, V. "Psychological and situational determinants of water conservation behavior: a structural model". Estudos de Psicologia 8 (2). 2003: 245-252.

Corral, V. “¿Mapas cognitivos o conductas ambientales?". Perspectivas sobre el cognitivismo en Psicología. Díaz, E. y Anaya, M. (coords.). México. UNAMIztacala, 2003a: 37-79.

Corral, V. "Determinantes psicológicos o situacionais do comportamento de conservaçào de àgua: um modelo estructural". Estudos de Psicologia 8. 2003b: 245-252.

Corral, V. "Situational and personal determinants of waste control practices in Northern Mexico: a study of reuse and recycling behaviors". Recourses, Conservation \& Recycling 39. 2003c: 265-281.

Corral, V. "Psicología ambiental interamericana". Medio Ambiente y Comportamiento Humano 7 (1). 2006: 1-5. 
Corral, V.; Bechtel, R.; Armendáriz, L. I. y Esquer, A. B. "La estructura de las creencias ambientales en universitarios mexicanos: el nuevo paradigma ambiental". Revista Mexicana de Psicología 14. 1997: 173-181.

Corral, V.; Bonnes, G.; Tapia, C.; Fraijo, B.; Frías, M. y Carrus, G. "Correlates of pro-sustainability orientation: the affinity towards diversity". Journal of Environment Psychology 29. 2009: 34-43.

Corral, V.; Carrus, G.; Bonnes, M.; Moser, G. y Sinha, J. "Environment beliefs and endorsement of sustainable development principles in water conservation". Environment and Behavior 40 (5). 2008: 703-725.

Corral, V. y Encinas, L. "Variables disposicionales, situacionales $y$ demográficas en el reciclaje de metal y papel". Medio Ambiente y Comportamiento Humano 2. 2001: 1-19.

Corral, V.; Fraijo, B. y Pinheiro, J. "Sustainable behavior and time perspective: present, past, and future orientations and their relationships with water conservation behavior". Revista Interamericana de Psicología 40 (2). 2006: 139-147.

Corral, V.; Fraijo, B. y Tapia, C. "Propensiones psicológicas en niños de sexto grado de primaria. Validez de un instrumento". Anuario de Investigaciones Educativas 7. 2004: 31-44.

Corral, V. y Frías, M. "Personal normative beliefs, antisocial behavior, and residential water conservation". Environment and Behavior 38. 2006: 406-421.

Corral, V.; Frías, M. y González, D. "On the relationship between antisocial and antienvironmental behaviors: an empirical study". Population and Environment 24. 2001: 273-286.

Corral, V.; Frías, M. y González, D. "Percepción de riesgos, conducta proambiental $y$ variables demográficas en una comunidad de Sonora, México". Región y Sociedad 26. 2003: 49-72.

Corral, V.; Frías, M.; Fraijo, B. y Tapia, C. "Rasgos de la conducta antisocial como correlatos del actuar anti y proambiental". Medio Ambiente $y$ Comportamiento Humano 7 (1). 2006 : 89-103.

Corral, V. y Obregón, F. J. "Modelos predictores del comportamiento proambientalista". Revista Sonorense de Psicología 6. 1992: 5-14.

Corral, V. y Pinheiro, J. "Condicoes para o estudo do comportamento próambiental”. Estudos de Psicología. 4. 1999: 7-22.

Corral, V. y Pinheiro, J. "Aproximaciones al estudio de la conducta sustentable". Medio Ambiente y Comportamiento Humano 5 (1-2). 2004: 1-26.

Corral, V.; Tapia, C.; Fraijo, B.; Mireles, J. y Márquez, P. "Orientación a la sustentabilidad como determinante de los estilos de vida sustentables. Un estudio con una muestra mexicana". Revista Mexicana de Psicología 25 (2). 2008: 313-327.

Corral, V.; Varela, C. y González, D. "Una taxonomía funcional de competencias proambientales". La Psicología Social en México 9. 2002: 592-597.

Corral, V. y Zaragoza, F. "Bases sociodemográficas y psicológicas de la conducta de reutilización: un modelo estructural". Medio Ambiente $y$ Comportamiento Humano 1. 2000: 9-29.

Espinosa, G.; Orduña, V. y Corral, V. "Modelamiento estructural de las competencias proambientales para el ahorro de agua”. La Psicología Social en México 9. 2002: 605-610.

Frías, M.; Martín, A. y Corral, V. "Análisis de factores que influyen en el desarrollo de normas ambientales $y$ en la conducta anti-ecológica". Revista Interamericana de Psicología 43 (2). 2009: 309-322. 
Hernández, B.; Corral, V.; Hess, S. y Suárez, E. "Sistemas de creencias ambientales: un análisis multimuestra de estructuras factoriales”. Estudios de Psicología 22. 2001: 53-64.

Kallis, G.; Ray, I.; Fulton, J. y McMahon, J. "Public versus private: does it matter for water conservation? Insights from California”. Environment Management 45 (1). 2010: 177-191.

Kline, R. Principles and practice of structural equations modelling. New York: Guilford Press, 1998.

Kline, R. "Reverse arrow dynamics. Formative measurement and feedback loops". Structural equation modeling: a second course. Hancock, G. y Mueller, R. (coords.). Greenwood, CT. Information Age Publishing Inc., 2006: 43-68.

Levy, J. "Modelización y análisis con ecuaciones estructurales". Análisis multivariable para las ciencias sociales. Levy, J. y Varela, J. (coords.). Madrid. Pearson Prentice Hall, 2003: 767-814.

Marandu, E.; Moeti, N. y Haika, J. "Predicting residential water conservation using the Theory of Reasoned Action". Journal of Communication 1 (2). 2010: 87-100.

March, H. y Sauri, D. "What lies behind domestic water use? A review essay on the drivers of domestic water consumption". Boletín de la Asociación de Geógrafos Españoles (AGEN) 50. 2009: 297-314.

Martínez, J. "La estructura de la responsabilidad ambiental hacia la conservación de agua potable en jóvenes estudiantes". Medio Ambiente y Comportamiento Humano 5. 2004: 115-132.

McFarie, B. y Hunt, L. "Environmental activism in the forest sector. Social psychological, social-cultural and contextual effects". Environment and Behavior 38. 2006: 266-285.

Milfont, T. y Duckitt, J. "The structure of environmental attitudes: a first and second cader confirmatory factor analysis". Journal of Environmental Psychology 24. 2004: 289-303.

Milfont, T. y Duckitt, J. "Preservation and utilization the structure of environmental attitudes". Medio Ambiente y Comportamiento Humano 7. 2006: 29-50.

Milfont, T. y Gouveia, V. "Time perspective and values: an exploratory study of their relations to environmental attitudes". Journal of Environmental Psychology 26. 2006: 72-82.

Mondéjar, J.; Cordente, M.; Meseguer, M. y Gázquez, J. "Environmental behavior and water saving in spanish housing". International Journal Environment Research 5 (1). 2011: 1-10.

Obregón, F. y Zaragoza, F. "La relación de tradición y modernidad con las creencias ambientales". Revista Sonorense de Psicología 14. 2000: 63-71.

Pato, C. y Tamayo, A. "Valores, creencias ambientales y comportamiento ecológico del activismo". Medio Ambiente y Comportamiento Humano 7 (1). 2006: 51-66.

Pato, C.; Ross, M. y Tamayo, A. "Creencias y comportamiento ecológico: un estudio empírico con estudiantes brasileños". Medio Ambiente y Comportamiento Humano 6 (1). 2005: 5-22.

Pato, C.; Tróccoli, B. y Tamayo, A. "Values and ecological behavior: an empirical study of Brazilians students". 17th Conference of IAPS. 2002.

Rusell, S. y Fielding, K. "Water demand management research: a psychological perspective". Water Resources Research 46. 2010: 1-12.

Syme, G.; Shao, Q.; Po, M. y Campbell, E. "Predicting and understanding home garden water use". Landscape and Urban Planning 68. 2004: 121-128.

Tal, I.; Hill, D.; Figueredo, A.; Frías, M. y Corral, V. "An evolutionary approach to explaning water conservation behavior". Medio Ambiente y Comportamiento Humano 7. 2006: 7-27. 
Thompson, S. C. y Stoutmeyer, K. "Water use and commons dilemma: the effects of education that focuses on long-term consequences and individual action". Environment and Behavior 23. 1991: 314-333.

Valenzuela, B.; Corral, V.; Quijada, A.; Griego, T.; Ocaña, D. y Contreras, C. "Predictores disposicionales del ahorro de agua: austeridad, altruismo y propensión al futuro". La Psicología Social en México 10. 2004: 527-532.

Zhao, R. y Chen, S. "Fuzzy pricing for urban water resources: model construction and application". Journal of Environment Management 8. 2008: 458-466.

Fecha de ingreso: $24 / 04 / 2012$ Fecha de aprobación: 14/12/2012 